\title{
Nonparametric specification testing via the trinity of tests
}

\author{
Abhimanyu Gupta *† \\ Department of Economics \\ University of Essex
}

November 20, 2017

\begin{abstract}
Tests are developed for inference on a parameter vector whose dimension grows slowly with sample size. The statistics are based on the Lagrange Multiplier, Wald and (pseudo) Likelihood Ratio principles, admit standard normal asymptotic distributions under the null and are straightforward to compute. They are shown to be consistent and possessing non-trivial power against local alternatives. The settings considered include multiple linear regression, panel data models with fixed effects and spatial autoregressions. When a nonparametric regression function is estimated by series we use our statistics to propose specification tests, and in semiparametric adaptive estimation we provide a test for correct error distribution specification. These tests are nonparametric but handled in practice with parametric techniques. A Monte Carlo study suggests that our tests perform well in finite samples. Two empirical examples use them to test for correct shape of an electricity distribution cost function and linearity and equality of Engel curves.
\end{abstract}

JEL classifications: C12, C14, C31, C33

Keywords: Increasingly many parameters; central limit theorem; Lagrange Multiplier test; Wald test; Likelihood Ratio test; nonparametric specification testing; series estimation; adaptive estimation; spatial autoregression

${ }^{*}$ I thank three anonymous referees for many insightful comments that led to a much improved paper, including a correction. I am also grateful for very helpful comments by Svetlana Bryzgalova, Marcus Chambers, Valentina Corradi, Tatiana Komarova, Federico Martellosio, Peter Phillips, Peter Robinson, Myunghwan Seo, João Santos Silva, Shruti Sinha, Sorawoot Srisuma, Luke Taylor and audiences at the University of Southampton, University of Surrey, IISA Annual Conference 2015 (Pune), Indian Statistical Institute (Delhi), University of Liverpool, Shanghai Jiao Tong University, ESEM 2016 (Geneva) and Seoul National University. I also thank Adonis Yatchew for permission to use the data employed in the empirical examples.

${ }^{\dagger}$ Email: a.gupta@essex.ac.uk, Telephone: +44-1206872 597 


\section{Introduction}

Many statistical models are parameterized by vectors that increase in dimension with sample size, making the study of asymptotic properties of estimates a nonparametric problem. We are concerned with inference on such growing parameter vectors in these type of models. Our tests statistics have desirable asymptotic properties in such settings and are easy to compute using standard formulae and software. We show that they can be applied to a wide variety of problems, including panel data models, spatial autoregressive models, and specification testing in nonparametric regression and adaptive estimation. Throughout the paper we will consider only cases where the parameter space grows slowly with sample size, as opposed to models in which the number of parameters exceeds or grows like the sample size.

Inference rules are complicated in increasing dimension settings by the fact that while usual Lagrange Multiplier (LM), Wald or pseudo Likelihood Ratio (LR) test statistics for $q$ (fixed) restrictions have an asymptotic $\chi_{q}^{2}$ distribution under the null hypothesis (under suitable regularity conditions), if in fact $q \rightarrow \infty$ with sample size this limit distribution no longer holds. However, as in previous literature (e.g. de Jong and Bierens (1994), Hong and White (1995)), we are motivated by the well-known fact that

$$
\frac{\chi_{q}^{2}-q}{2^{\frac{1}{2}} q^{\frac{1}{2}}} \stackrel{d}{\longrightarrow} \mathcal{N}(0,1), \text { as } q \rightarrow \infty
$$

and will justify asymptotic properties for such standardized test statistics.

The literature on multiple regression with increasingly many parameters dates back at least to Huber (1973) but the sequence of experiments considered by Le Cam (1960) is an even earlier reference to models that evolve with sample size, see van der Vaart (2002) for a discussion. Portnoy $(1984,1985)$ studied more general $M$-estimates of linear regression with growing dimension, and Andrews (1985) also stressed that frequently the choice of regressors is motivated more by degrees of freedom constraints than actual economic theory, hence the appeal of a theory that permits the number of regressors to be related to sample size. While practitioners can adopt an attitude that permits precise estimation of larger models with more data, arrival at a parsimonious model requires rules of inference. Testing of approximate models also requires such rules, e.g. Berk (1974) considered time series autoregressions with increasing dimension while Robinson (1979) studied models with increasing dimension as approximations to infinite distributed lag systems. More recently, Robinson (2003) examined the problem of estimating the parameters of a single equation 
in a system of increasingly many equations. A very recent development is interest in spatial autoregressions (SAR) with increasing dimension, treated in Gupta and Robinson (2015, 2018), the latter paper permitting a nonlinear regression component of increasing dimension. They point out that SAR models can sometimes give rise to increasing parameter asymptotics quite naturally.

An important context in which models of increasing dimension are estimated is series estimation of nonparametric regression, where a nonparametric regression function is approximated by a growing number of basis functions whose coefficients need to be estimated, see e.g. Andrews (1991), Newey (1997). These models and such an estimation strategy offer an attractive role for inferential rules based on increasingly many restrictions. One use, explored for instance by Eubank and Spiegelman (1990), Wooldridge (1992), Hong and White (1995) and Donald, Imbens, and Newey (2003) is to test regression function specification, providing an alternative to kernel based nonparametric specification testing, see e.g. Fan and Li (1996), Zheng (1996) and Lavergne and Vuong (2000). We also provide such tests, but our general results have some differences. We avoid normality of disturbances (Eubank and Spiegelman (1990)), sample splitting (Yatchew (1992)) and do not not impose that the data be generated from an iid process (Wooldridge (1992), Hong and White (1995), Donald et al. (2003)). Additionally we base our test statistics on the trinity of tests, which can provide as simple, or even simpler, inference than the sums of squares based statistics of Hong and White (1995). Our approach can handle a variety of interesting cases. These include, but are not limited to, tests of significance of nonparametric regressions, tests of linearity against a nonparametric alternative and tests of a partially linear model against a fully nonparametric alternative. Hong and White (1995) offer more alternatives to test against, indeed even nonlinear parametric alternatives, but unlike that paper our focus is not on series based nonparametric specification alone. Another use that we propose is in testing the unknown specification of the error distribution in the semiparametric series-based adaptive estimation techniques employed by, e.g., Beran (1976), Newey (1988) and Robinson (2005, 2010). The motivation of our tests is rather different from work such as Chen and Pouzo (2015), which focuses on inference on a functional in a conditional moment model with an infinite dimensional parameter that is not of primary interest, or Shen and Shi (2005), wherein there is an infinite dimensional nuisance parameter and interest lies in known functionals.

Simplification of nonparametric and semiparametric inference is an important issue in practical work. A recent paper by Ackerberg, Chen, and Hahn (2012) notes that the 
complicated nature of semiparametric methods tends to make practitioners reluctant to use them. They stress cases in which the practitioner can effectively ignore the semiparametric nature of certain econometric problems and simply use formulae derived for parametric cases, thus increasing considerably the appeal of semiparametric methods. Our approach to nonparametric and semiparametric testing is in the same spirit. Effectively the tests of specification boil down to simple inference on coefficients in linear regression models with standard testing principles. We hope that this simplicity adds to the toolkit of 'parametriclike' procedures in problems that are not fully parametric.

In Section 2 we introduce the setup as well as two examples. Section 3.1 defines the test statistics and introduces their desirable property that we seek sufficient conditions for. Section 3.2 contains the asymptotic theory, the conditions of which we illustrate in our examples. Section 4 uses these ideas to propose a simple specification test for use in nonparametric regression, while Section 5 introduces a test for error distribution specification in adaptive estimation. Section 6 contains a Monte Carlo study of finite sample performance, also discussing some implementation issues. In Section 7 we use our tests to determine the shape of a Canadian electricity distribution cost function and to test for linearity and equality of Engel curves from South African data. Section 8 concludes, briefly discussing heteroskedasticity robust versions of our tests. Proofs are in appendices.

\section{Setup}

We observe a vector $w_{i} \equiv w_{i n}, i=1, \ldots, n$, of dimension at least $s+1$, with $s$ a positive integer. The triangular array setup permits sufficient generality to cover many important cases. The unknown true parameter vector $\theta_{0} \in \mathbb{R}^{s}$, is estimated by

$$
\tilde{\theta}=\underset{\theta \in \Theta}{\arg \min } \mathcal{Q}_{n}\left(w_{1}, \ldots, w_{n} ; \theta\right)
$$

where $\Theta \subseteq \mathbb{R}^{s}$. The dimension $s$ of $\theta_{0}$ is regarded as $n$-dependent, with $s \rightarrow \infty$ as $n \rightarrow \infty$ although explicit reference to this dependence is suppressed for notational convenience. We also suppress reference to the observations $w=\left(w_{1}, \ldots, w_{n}\right)$ in the objective function, writing it as $\mathcal{Q}_{n}(\theta)$. The sequence of true values $\theta_{0 n}$ satisfies $\theta_{0}=\arg \min _{\theta \in \Theta} \mathbb{E}\left\{\mathcal{Q}_{n}(\theta)\right\}$, where we suppress the $n$ subscript and $\mathbb{E}\left\{\mathcal{Q}_{n}(\theta)\right\}$ is a 'population' counterpart of $\mathcal{Q}_{n}(\theta)$ for a given sample size $n$. An example of a population counterpart is given in Example I below. 
The parameter $\theta_{0}$ is identified with respect to $\left(\mathcal{Q}_{n}(\theta), \Theta\right)$ if there is no $\theta^{\dagger} \in \Theta \backslash\left\{\theta_{0}\right\}$ such that $\mathbb{E}\left\{\mathcal{Q}_{n}\left(\theta_{0}\right)\right\}=\mathbb{E}\left\{\mathcal{Q}_{n}\left(\theta^{\dagger}\right)\right\}$. Particular conditions guaranteeing the latter vary according to the particular econometric model under consideration. Generally for implicitly defined models a condition of the type $\mathbb{E}\left\{\mathcal{Q}_{n}(\theta)\right\}-\mathbb{E}\left\{\mathcal{Q}_{n}\left(\theta_{0}\right)\right\}>0$ for all $\theta \in \Theta \backslash\left\{\theta_{0}\right\}$ is checked for global identification, with suitable conditions imposed on model primitives to satisfy this. Given a sample of size $n$, for models with closed form solutions for $\tilde{\theta}$ this typically reduces to non-singularity of certain second moment matrices, i.e. nonmulticollinearity conditions.

Assumption $1 . \mathcal{Q}_{n}(\theta)$ is convex and twice differentiable in $\theta \in \Theta$, for all sufficiently large $n$.

In situations where $\tilde{\theta}$ is implicitly defined $\Theta$ may be a prescribed compact set, which needs to be constrained to remain of bounded volume due to its increasing dimension. For instance Gupta and Robinson (2018) choose a Cartesian product of intervals of unit length. Assumption 1 ensures that $\tilde{\theta}$ exists for sufficiently large $n$, and allows us to define

$$
g_{n}(\theta)=\frac{\partial \mathcal{Q}_{n}(\theta)}{\partial \theta}, \quad H_{n}(\theta)=\frac{\partial^{2} \mathcal{Q}_{n}(\theta)}{\partial \theta \partial \theta^{\prime}}
$$

with primes denoting transposition.

We now proceed to the setup of the general testing problem. Split the parameter vector as $\theta=\left(\theta_{1}^{\prime}, \theta_{2}^{\prime}\right)^{\prime}$ where $\theta_{1}$ is $q \times 1$, with $q \leq s$ and $q \rightarrow \infty$ as $n \rightarrow \infty$, and $\theta_{2}$ is $(s-q) \times 1$. For a generic symmetric, non-negative definite matrix $J$, define by $\bar{\eta}(J)(\underline{\eta}(J))$ its largest (smallest) eigenvalue, and define $\|K\|=\left\{\bar{\eta}\left(K^{\prime} K\right)\right\}^{\frac{1}{2}}$ for any generic matrix $K$. If $K$ is a vector, then $\|K\|$ is simply the Euclidean norm. We are interested in testing hypotheses of the type

$$
\begin{array}{lll}
H_{0 n} \equiv H_{0} & : & \left\|\theta_{10}\right\|=0 \\
H_{1 n} \equiv H_{1} & : & \left\|\theta_{10}\right\| \neq 0
\end{array}
$$

where $\theta_{0}=\left(\theta_{10}^{\prime}, \theta_{20}^{\prime}\right)^{\prime}$ is the true parameter value. As usual $\theta$ can be a transformation of some underlying parameters, so that the above formulation is general enough to cover an increasing number of linear restrictions on model parameters. We also denote the parameter space under the restriction $(2.3)$ as $\Theta^{0}$ and define the restricted estimate

$$
\hat{\theta}=\underset{\theta \in \Theta^{0}}{\arg \min } \mathcal{Q}_{n}(\theta) .
$$


In the sequel we will assume that $\theta_{0}$ lies in the interior of $\Theta$.

Example I. Inference in regression models with increasing dimension.

Portnoy $(1984,1985)$ considers

$$
y_{n}=X_{n} \beta+v
$$

where throughout the paper $y \equiv y_{n}$ denotes an $n \times 1$ vector of observations, $X \equiv X_{n}$ an $n \times k$ matrix of exogenous explanatory variables, with $k \rightarrow \infty$ as $n \rightarrow \infty$, and $v$ an unobserved $n \times 1$ disturbance vector with iid elements $v_{i}$ having mean zero and unit variance. He takes

$$
\mathcal{Q}_{n}^{M}(\beta)=\frac{1}{n} \sum_{i=1}^{n} \psi\left(y_{i}-x_{i}^{\prime} \beta\right),
$$

where $x_{i} \equiv x_{i, n}$ is the $i$-th column of $X^{\prime}, y_{i} \equiv y_{i, n}$ is the $i$-th element of $y$ and $\psi: \mathbb{R} \rightarrow \mathbb{R}$. Thus $s=k$ and $w_{i}=\left(y_{i}, x_{i}^{\prime}\right)^{\prime}$. The population counterpart of $(2.7)$ is $\mathbb{E}\left\{\psi\left(Y-X_{i}^{\prime} \beta\right)\right\}$, where $\left(Y, X_{i}^{\prime}\right)^{\prime}$ denotes the random variables from which the sample $\left(y_{i}, x_{i}^{\prime}\right)^{\prime}$ is drawn. The ordinary least squares (OLS) estimate is obtained by taking $\psi(x)=x^{2} / 2$, and we maintain this case in this paper. Panel data models with fixed effects can also be accommodated. Consider a balanced panel with $N$ observations in each of $T$ individual panels, so that $n=N T$, with $N, T \rightarrow \infty$ together. Let $y_{t N}$ be the $N \times 1$ vector of observations on the dependent variable for the $t$-th panel, where $t$ may correspond to a time period or a more general spatial unit like a school, village or district. Also let $X_{1, t N}$ and $X_{2, N}$ be $N \times k_{1}$ and $N \times k_{2}$ matrices of exogenous regressors respectively. $X_{1, t N}$ contains panel-varying regressors while $X_{2, N}$ does not. Consider the model

$$
y_{t N}=\iota_{N} \alpha_{t}+X_{1, t N} \beta_{1}+X_{2, N} \beta_{2 t}+v_{t N}, \quad t=1, \ldots, T
$$

where $v_{t N}$ is the $N \times 1$ vector of disturbances for each panel, formed of iid components, and $\iota_{N}$ is the $N \times 1$ vector of ones. The $\alpha_{t}, t=1, \ldots, T$, are scalar fixed effect parameters and $\beta_{1}$ is a $k_{1} \times 1$ panel-invariant parameter vector, whereas $\beta_{2 t}$ is a $k_{2} \times 1$ parameter vector that can vary with $t$, so $X_{2, N}$ may be thought of as controlling for 'quasi' fixed-effects. Denote $y_{n}=\left(y_{1 n}^{\prime}, \ldots, y_{T n}^{\prime}\right)^{\prime}, X_{1, n}=\left(X_{1,1 n}^{\prime}, \ldots, X_{1, T n}^{\prime}\right)^{\prime}, v=\left(v_{1 n}^{\prime}, \ldots, v_{T n}^{\prime}\right)^{\prime}, \alpha=\left(\alpha_{1}, \ldots, \alpha_{T}\right)^{\prime}$ and $\beta_{2}=\left(\beta_{21}^{\prime}, \ldots, \beta_{2 T}^{\prime}\right)^{\prime}$. Writing $I_{T}$ for the $T \times T$ identity matrix we can then stack (2.8) to obtain

$$
y_{n}=\left(I_{T} \otimes \iota_{N}\right) \alpha+X_{1, n} \beta_{1}+\left(I_{T} \otimes X_{2, N}\right) \beta_{2}+v,
$$

which can be written like (2.6) by taking $X_{n}=\left(I_{T} \otimes \iota_{N}, X_{1, n}, I_{T} \otimes X_{2, N}\right)$ and $\beta=$ $\left(\alpha^{\prime}, \beta_{1}^{\prime}, \beta_{2}^{\prime}\right)^{\prime}$, implying $s=k_{1}+T\left(k_{2}+1\right)$. Again we may dispense with $n$ subscripting 
for brevity.

Our theory will permit inference on subsets of $\beta$ of increasing dimension. A question of practical interest is whether the fixed effects $\alpha_{t}$ in (2.8) are zero, or more generally if they are equal. Thus we may be interested in testing:

$$
H_{0}:\left\|\alpha-c \iota_{T}\right\|=0, c \text { known scalar. }
$$

Example II. Inference in spatial autoregressive (SAR) models with increasing dimension. The SAR model was introduced by Cliff and Ord (1973) and has seen heavy use since in modelling of spatial correlation and dependence. For a given set of known weight matrices $W_{i n}, i=1, \ldots, p$, whose elements are a measure of economic (not necessarily geographic) distance between units, $y_{n}$ is modelled as

$$
y_{n}=\sum_{i=1}^{p} \lambda_{i} W_{i n} y_{n}+X_{n} \beta+v
$$

The $\lambda_{i}$ capture spatial dependence between units. We write $R_{n}=\left[W_{1 n} y_{n}, \ldots, W_{p n} y_{n}\right]$ and $\theta=\left(\lambda^{\prime}, \beta^{\prime}\right)^{\prime}$, where $\lambda$ has $i$-th element $\lambda_{i}, i=1, \ldots, p$, so $s=p+k$. $X_{n}$ may also contain spatial lags of regressors, so its columns need not be independent or identically distributed in general. The elements of the $W_{i n}$ themselves are usually normalized in some way with a normalization factor that depends on $n$, indeed some normalization is necessary to identify the $\lambda_{i}$. Thus the triangular array aspect here is not merely a technical generalization but an important feature of the model. Each column of $R_{n}$ is endogenous, so OLS estimation of $\theta$ does not work in general but Lee (2002) showed that for $p=1$ consistency follows if the elements of $W_{\text {in }}$ are $\mathscr{O}\left(h^{-1}\right)$ for some $h \rightarrow \infty$ such that $h=o(n)$, and asymptotic normality and efficiency if also $n^{\frac{1}{2}}=o(h)$. The asymptotic properties of the instrumental variables (IV) estimate were justified by Kelejian and Prucha (1998), while Lee (2004) presented a number of results for Gaussian (although Gaussianity is nowhere assumed) pseudo maximum likelihood estimates (PMLE). Gupta and Robinson (2015, 2018) have introduced an increasing dimension $(p, k \rightarrow \infty)$ version of higher-order models such as (2.11) that contain more than one spatial lag of $y_{n}$. They consider OLS, IV and PML estimates of $\theta$. The latter works also when $\beta=0$, unlike the first two, which require the presence of at least one non-intercept regressor. Let $Z_{n}$ be an $n \times r$ matrix of instruments, $r \geq p$, write $\mathscr{P}(J)=J\left(J^{\prime} J\right)^{-1} J^{\prime}$ for a matrix $J$ with full column rank and define $\hat{\theta}_{S A R}^{I V}$ and 
$\hat{\theta}_{S A R}^{O L S}$ as the $\theta$ minimizing

$$
\begin{aligned}
\mathcal{Q}_{n}^{S A R, I V}(\theta) & =\frac{1}{2 n}\left(y_{n}-\left[R_{n}, X_{n}\right] \theta\right)^{\prime} \mathscr{P}\left(\left[Z_{n}, X_{n}\right]\right)\left(y_{n}-\left[R_{n}, X_{n}\right] \theta\right), \\
\mathcal{Q}_{n}^{S A R, O L S}(\theta) & =\frac{1}{2 n}\left(y_{n}-\left[R_{n}, X_{n}\right] \theta\right)^{\prime}\left(y_{n}-\left[R_{n}, X_{n}\right] \theta\right)
\end{aligned}
$$

respectively. Denoting $S_{n}(\lambda)=I_{n}-\sum_{i=1}^{p} \lambda_{i} W_{i n}$, the PMLE is based on the Gaussian likelihood

$$
\log (2 \pi)-2 n^{-1} \log \left|S_{n}(\lambda)\right|+n^{-1}\left\|S_{n}(\lambda) y_{n}-X_{n} \beta\right\|^{2}
$$

For given $\lambda,(2.14)$ is minimised with respect to $\beta$ by $\bar{\beta}(\lambda)=\left(X_{n}^{\prime} X_{n}\right)^{-1} X_{n}^{\prime} S_{n}(\lambda) y_{n}$. We define $\hat{\lambda}_{S A R}^{P M L}=\arg \min _{\lambda \in \Lambda} \mathcal{Q}_{n}^{S A R, P M L}(\lambda)$, with $\Lambda$ a compact set in $\mathbb{R}^{p}$, where

$$
\mathcal{Q}_{n}^{S A R, P M L}(\lambda)=n^{-1} \log \left|S_{n}^{-1}(\lambda) S_{n}^{-1 \prime}(\lambda)\right|+n^{-1} y_{n}^{\prime} S_{n}^{\prime}(\lambda)\left(I_{n}-\mathscr{P}\left(X_{n}\right)\right) S_{n}(\lambda) y_{n},
$$

The PMLE of $\beta_{0}$ is defined as $\bar{\beta}(\hat{\lambda}) \equiv \hat{\beta}_{S A R}^{P M L}$.

Gupta and Robinson $(2015,2018)$ stress cases motivated by Case $(1991,1992)$ in which the $W_{\text {in }}$ have a 'single non-zero diagonal block' structure. In such cases it is explicitly assumed that there are no spatial effects between units not in the same block, and it is reasonable to expect that the $\lambda_{i}$ vary across blocks. However there can be reasons (e.g. geographic or demographic) for practitioners to suspect that some of the $\lambda_{i}$ may be equal. Of particular interest is the case where all the $\lambda_{i}$ are equal, implying a simpler model in which $p=1$, and a model of fixed dimension if in fact $k$ is fixed. Denoting by $\lambda^{D}$ the $p(p-1) / 2$-dimensional vector with elements $\lambda_{i}-\lambda_{j}, i \neq j$ and $i, j=1, \ldots, p$, this kind of test can be captured in the null hypothesis

$$
H_{0}:\left\|\lambda^{D}\right\|=0
$$

or, if a common value can be hypothesized,

$$
H_{0}:\left\|\lambda-c \iota_{p}\right\|=0, c \text { known scalar. }
$$




\section{Trinity motivated tests}

\subsection{The statistics and a desirable property}

For any function $f(\cdot)$ we will write $f(\check{\theta}) \equiv \check{f}$ and $f\left(\theta_{0}\right) \equiv f$, where $\check{\theta}$ is a generic estimate of $\theta$, following this convention throughout the paper. Define the standardized LM, Wald and LR test statistics

$$
\begin{aligned}
\mathbf{L M}_{n} & =\frac{n \hat{g}_{1, n}^{\prime} \hat{H}_{n}^{11} \hat{g}_{1, n}-q}{2^{\frac{1}{2}} q^{\frac{1}{2}}}, \\
\mathbf{W}_{n} & =\frac{n \tilde{\theta}_{1}^{\prime}\left(\tilde{H}_{n}^{11}\right)^{-1} \tilde{\theta}_{1}-q}{2^{\frac{1}{2}} q^{\frac{1}{2}}}, \\
\mathbf{L R}_{n} & =\frac{2 n\left(\hat{\mathcal{Q}}_{n}-\tilde{\mathcal{Q}}_{n}\right)-q}{2^{\frac{1}{2}} q^{\frac{1}{2}}},
\end{aligned}
$$

with $\hat{g}_{n}=\left(\hat{g}_{1, n}^{\prime}, \hat{g}_{2, n}^{\prime}\right)^{\prime}$ and

$$
\hat{H}_{n}^{-1}=\left[\begin{array}{cc}
\hat{H}_{n}^{11} & \hat{H}_{n}^{12} \\
\hat{H}_{n}^{21} & \hat{H}_{n}^{22}
\end{array}\right]
$$

where $\hat{g}_{1, n}$ is $q \times 1, \hat{g}_{2, n}$ is $(s-q) \times 1, \hat{H}_{n}^{11}$ is $q \times q, \hat{H}_{n}^{12}$ is $q \times(s-q)$ and $\hat{H}_{n}^{22}$ is $(s-q) \times(s-q)$. This convention for partitioning is adopted throughout so we will denote

$$
J_{n}=\left[\begin{array}{ll}
J_{11, n} & J_{12, n} \\
J_{21, n} & J_{22, n}
\end{array}\right], J_{n}^{-1}=\left[\begin{array}{cc}
J_{n}^{11} & J_{n}^{12} \\
J_{n}^{21} & J_{n}^{22}
\end{array}\right]
$$

for a generic nonsingular $s \times s$ matrix $J_{n}$. LM tests have the favourable feature of requiring estimation of the model only under the null hypothesis, which yields a more parsimonious null model always. In the increasing parameter context sometimes even a finite dimensional null model may be implied, as in Example II. As usual the Wald statistic is based on the unrestricted estimates alone, while the LR statistic is based on both unrestricted and restricted estimates.

We seek to provide sufficient conditions for three desirable features of the test statistics defined above, encapsulated in the following definition.

Definition A sequence of random variables (test statistics) $\mathfrak{A}_{n}$ is said to have Property $\mathcal{C}$ if: 
C.1. Under $H_{0}$,

$$
\mathfrak{A}_{n} \stackrel{d}{\longrightarrow} \mathcal{N}(0,1), \text { as } n \rightarrow \infty
$$

C.2. Under $H_{1}$ and $q^{\frac{1}{4}} / n^{\frac{1}{2}} \longrightarrow 0$ as $n \rightarrow \infty$, for any $\varrho>0$,

$$
P\left(\left|\mathfrak{A}_{n}\right|>\varrho\right) \longrightarrow 1, \text { as } n \rightarrow \infty
$$

C.3. Define $H_{\ell 1} \equiv H_{\ell 1, n}: \theta_{\ell, 10}=\theta_{\ell, 10, n}=\delta_{n} q^{\frac{1}{4}} /\left(n \delta_{n}^{\prime} \Gamma_{n} \delta_{n}\right)^{\frac{1}{2}}$, where $\Gamma_{n}$ is a constant symmetric $q \times q$ matrix with $\underline{\lim }_{n \rightarrow \infty} \underline{\eta}\left(\Gamma_{n}\right)>0, \varlimsup_{n \rightarrow \infty} \bar{\eta}\left(\Gamma_{n}\right)<\infty$ and $\delta_{n}$ a constant $q \times 1$ vector such that $\left\|\delta_{n}\right\|=1$. Then, under the sequence of local alternatives $H_{\ell 1}$ and $q^{\frac{1}{4}} / n^{\frac{1}{2}} \longrightarrow 0$ as $n \rightarrow \infty$,

$$
\mathfrak{A}_{n} \stackrel{d}{\longrightarrow} \mathcal{N}\left(2^{-\frac{1}{2}}, 1\right) \text {, as } n \rightarrow \infty \text {. }
$$

The conditions specify the asymptotic distribution of $\mathfrak{A}_{n}$ under $H_{0}$, the consistency of the test based on $\mathfrak{A}_{n}$ as test statistic, and the fact that such a test can detect local alternatives at $\mathscr{O}\left(q^{\frac{1}{4}} / n^{\frac{1}{2}}\right)$ rate. The latter rate has also been found by de Jong and Bierens (1994).

\section{$3.2 \quad$ Asymptotic theory}

In this section we describe the asymptotic behaviour of the test statistics when $g_{n}$ asymptotically differs negligibly from a linear function of an $n \times 1$ unobservable disturbance vector $\epsilon$ with elements $\epsilon_{i}$. Let $C$ denote a generic constant, arbitrarily large but independent of $n$.

Assumption 2. The elements of $\epsilon$ are independent with zero mean, unit variance, finite third and fourth moments $\mu_{3}$ and $\mu_{4}$, and satisfy $\sup _{i \geq 1} \mathbb{E}\left|\epsilon_{i}\right|^{4+\chi} \leq C$, for some $\chi>0$.

Imposing unit variance simplifies our notation but is not restrictive as all results hold with independent homoskedastic disturbances, the latter simply adding another layer of derivations in the proofs and thus avoided here, but discussed in examples. Heteroskedasticity robustness is discussed in Section 8. Introduce an $n \times s$ matrix $M_{n}$ and the $s \times s$ constant and symmetric matrix $L_{n}=\mathbb{E}\left(n^{-1} M_{n}^{\prime} M_{n}\right)$ satisfying the following assumption:

Assumption 3. The elements of $M_{n}$ are independent of $\epsilon$, and there exists a sequence $m \equiv m_{n}$, divergent or bounded, such that their second moments are uniformly $\mathscr{O}\left(m^{2}\right)$. The eigenvalues of $L_{n}$ are such that $\overline{\lim }_{n \rightarrow \infty} \bar{\eta}\left(L_{n}\right)<\infty$ and $\underline{\lim }_{n \rightarrow \infty} \underline{\eta}\left(L_{n}\right)>0$. 
The condition on the elements of $M_{n}$ implies that these are uniformly $\mathscr{O}_{p}(m)$ and the rows of $M_{n}$ have uniformly $\mathscr{O}_{p}\left(s^{\frac{1}{2}} m\right)$ norm. The restrictions on the eigenvalues of $L_{n}$ are asymptotic 'no multicollinearity' and boundedness conditions of the type familiar from regression with increasing dimension. For generic matrices, vectors or scalars $J_{n}, K_{n}$ we denote $\Delta_{K}^{J}=J_{n}-K_{n}$ and for any symmetric matrix $J_{n}$ partitioned in the usual way, denote $\mathcal{Z}^{J}=\left[I_{q},-J_{12, n} J_{22, n}^{-1}\right]$. While both $\Delta_{K}^{J}$ and $\mathcal{Z}^{J}$ are $n$-dependent we avoid this in the notation to avoid excessive strain on the reader.

Assumption 4. The following rate condition holds:

$$
\begin{aligned}
& \frac{1}{q^{\frac{1}{2}}}\left\|\Delta_{n^{-1} M^{\prime} \epsilon}^{g}\right\|\left(n\left\|\Delta_{n^{-1} M^{\prime} \epsilon}^{g}\right\|+\left\|M_{n}^{\prime} \epsilon\right\|\right)+\left\|\Delta_{H}^{H^{*}}\right\|+\left\|\Delta_{n^{-1} M^{\prime} M}^{H}\right\|+\left\|\Delta_{L}^{n^{-1} M^{\prime} M}\right\| \\
& +\frac{1}{q^{\frac{1}{2}} n}\left\|M_{n}^{\prime} \epsilon\right\|^{2}\left(\left\|\Delta_{H}^{H^{*}}\right\|+\left\|\Delta_{n^{-1} M^{\prime} M}^{H}\right\|+\left\|\Delta_{L}^{n^{-1} M^{\prime} M}\right\|\right)=o_{p}(1), \text { as } n \rightarrow \infty,
\end{aligned}
$$

for any $\theta^{*}$ satisfying $\left\|\Delta_{\theta_{0}}^{\theta^{*}}\right\| \leq\left\|\Delta_{\theta_{0}}^{\hat{\theta}}\right\|$.

Theorem 3.1. Suppose that $H_{0}$, Assumptions 1, 2, 3 and 4 hold. Then

$$
\mathbf{L M}_{n}-\frac{\epsilon^{\prime} \mathcal{M}_{n} \epsilon-q}{2^{\frac{1}{2}} q^{\frac{1}{2}}}=o_{p}(1), \text { as } n \rightarrow \infty
$$

where $\mathcal{M}_{n}=n^{-1} M_{n} \mathcal{Z}^{n^{-1} M^{\prime} M}\left(n^{-1} M_{n}^{\prime} M_{n}\right)^{11} \mathcal{Z}^{n^{-1} M^{\prime} M} M_{n}^{\prime}$

Condition (3.4) looks intimidating but is essentially about some weak laws of large numbers holding. We discuss it for special cases in Remarks 2 and 3 below. For a generic $s \times s$ matrix $J_{n}$, define $\Sigma^{J}$ (note again the avoidance of subscripting with $n$ ) as the $s \times s$ matrix with bottom-right $(s-q) \times(s-q)$ block $J_{22, n}^{-1}$ and all other entries zero.

Theorem 3.2. Suppose that $H_{0}$, Assumptions 1, 2, 3 and 4 hold, with (3.4) now holding for any $\theta^{*}$ satisfying $\left\|\Delta_{\theta_{0}}^{\theta^{*}}\right\| \leq\left\|\Delta_{\theta_{0}}^{\hat{\theta}}\right\|$ or $\left\|\Delta_{\theta_{0}}^{\theta^{*}}\right\| \leq\left\|\Delta_{\theta_{0}}^{\tilde{\theta}}\right\|$. Then

$$
\begin{gathered}
\mathbf{W}_{n}-\frac{n^{-1} \epsilon^{\prime} M_{n}\left[\left(n^{-1} M_{n}^{\prime} M_{n}\right)^{-1}-\Sigma^{n^{-1} M^{\prime} M}\right] M_{n}^{\prime} \epsilon-q}{2^{\frac{1}{2}} q^{\frac{1}{2}}}=o_{p}(1) \text {, as } n \rightarrow \infty, \\
\mathbf{L R}_{n}-\frac{n^{-1} \epsilon^{\prime} M_{n}\left[\left(n^{-1} M_{n}^{\prime} M_{n}\right)^{-1}-\Sigma^{n^{-1} M^{\prime} M}\right] M_{n}^{\prime} \epsilon-q}{2^{\frac{1}{2}} q^{\frac{1}{2}}}=o_{p}(1) \text {, as } n \rightarrow \infty .
\end{gathered}
$$


It can be easily shown using the properties of partitioned matrices that

$$
n^{-1} M_{n}\left[\left(n^{-1} M_{n}^{\prime} M_{n}\right)^{-1}-\Sigma^{n^{-1} M^{\prime} M}\right] M_{n}^{\prime}=\mathcal{M}_{n},
$$

thus anticipating the asymptotic equivalence of the trinity of tests that we will prove below, and also familiar from the fixed dimension case.

Assumption 5. The sequences $q, s$ and $m$ are functions of $n$ satisfying

$$
\frac{1}{q^{1+\frac{\chi}{4}}}\left(\frac{\left(s^{\frac{1}{2}} m\right)^{2+\frac{\chi}{2}}}{n^{\frac{\chi}{4}}}+\frac{\left(s^{\frac{1}{2}} m\right)^{4+\chi}}{n^{1+\frac{\chi}{2}}}\right) \longrightarrow 0, \text { as } n \rightarrow \infty .
$$

In Theorems 3.1 and 3.2 we showed that the trio of test statistics may be approximated by a quadratic form in $\epsilon$. The next two theorems state the asymptotic distribution of these quadratic forms under suitable conditions.

Theorem 3.3. Suppose that $H_{0}$, Assumptions 1, 2, 3 and 5 hold. Then

$$
\frac{\epsilon^{\prime} \mathcal{M}_{n} \epsilon-q}{2^{\frac{1}{2}} q^{\frac{1}{2}}} \stackrel{d}{\longrightarrow} \mathcal{N}(0,1) \text {, as } n \rightarrow \infty
$$

The proof is in Appendix A and employs a martingale CLT of Scott (1973) as opposed to the $U$-statistic CLTs used in earlier literature, cf. e.g. de Jong and Bierens (1994), Hong and White (1995). For models with uniformly bounded constant $M_{n}$ elements we have $m \equiv 1$, so Condition (3.7) simplifies to

$$
\left(\frac{s}{q}\right)^{1+\frac{\chi}{4}} \frac{1}{n^{\frac{\chi}{4}}}\left(1+\left(\frac{s}{n}\right)^{1+\frac{\chi}{4}}\right) \longrightarrow 0, \text { as } n \rightarrow \infty,
$$

and is therefore satisfied if $s / q=\mathscr{O}(1)$ and $s / n \rightarrow 0$ as $n \rightarrow \infty$. In the panel data case of Example I, $s=k_{1}+T\left(k_{2}+1\right)$ and $n=N T$, while if we are interested in testing (2.10) then $q=T$. Thus (3.8) holds if $k_{1} / T+k_{2}$ is bounded and $k_{1} / N T+k_{2} / N \rightarrow 0$ as $N, T \rightarrow \infty$. The latter condition is implied by the former.

Theorem 3.4. Suppose that $H_{0}$, Assumptions 1, 2, 3 and 5 hold. Then

$$
\frac{n^{-1} \epsilon^{\prime} M_{n}\left[\left(n^{-1} M_{n}^{\prime} M_{n}\right)^{-1}-\Sigma^{n^{-1} M^{\prime} M}\right] M_{n}^{\prime} \epsilon-q}{2^{\frac{1}{2}} q^{\frac{1}{2}}} \stackrel{d}{\longrightarrow} \mathcal{N}(0,1) \text {, as } n \rightarrow \infty \text {. }
$$


Remark 1. In all of the theorems above, if $g_{n}(\theta)$ is linear in $\theta$ the part of the rate condition (3.4) relating to $\left\|\Delta_{H}^{H^{*}}\right\|$ is not needed. Indeed, in this case there will be a closed form for $\hat{\theta}$ and $\tilde{\theta}$, and the second derivative will not depend on $\theta$. A typical requirement for asymptotic normality proofs of implicitly defined estimates is $\left\|\hat{\theta}-\theta_{0}\right\|=o_{p}(1)$, which is covered in (3.4) by the negligibility conditions on $\left\|\Delta_{H}^{H^{*}}\right\|$. If estimates are of closed form then $\left\|n^{-1} M_{n}^{\prime} \epsilon\right\|=o_{p}(1)$ and $\left\|\Delta_{L}^{n^{-1} M^{\prime} M}\right\|=o_{p}(1)$ together with $\underline{\lim }_{n \rightarrow \infty} \underline{\eta}\left(L_{n}\right)>0$ suffice for $\left\|\hat{\theta}-\theta_{0}\right\|=o_{p}(1)$. As these are all conditions imposed in (3.4) consistency (in norm) of the estimates is guaranteed.

The following theorem records sufficient conditions for Property $\mathcal{C}$ to hold.

Theorem 3.5. (i) Under the conditions of Theorems 3.1 and 3.3, $\mathbf{L M}_{n}$ has Property $\mathcal{C}$.

(ii) Under the conditions of Theorems 3.2 and 3.4, $\mathbf{W}_{n}$ and $\mathbf{L R}_{n}$ have Property $\mathcal{C}$.

The appropriate choices of $\Gamma_{n}$ in $\mathcal{C} .3$ are found in the proof, while $\delta_{n}$ would typically be the vector with unity in the position corresponding to the direction of departure from $H_{0}$. In the remarks that follow we focus only on rate conditions, assuming the identification conditions to hold.

Remark 2. In Example I, $g_{n}=n^{-1} M_{n}^{\prime} v, M_{n}=X_{n}$ and $H_{n}=n^{-1} X_{n}^{\prime} X_{n}$. If the $x_{i}$ are iid with finite fourth moment $m$ is a bounded sequence, $L_{n}=\mathbb{E}\left(x_{i} x_{i}^{\prime}\right),\left\|M^{\prime} v\right\|=\mathscr{O}_{p}\left((k n)^{\frac{1}{2}}\right)$ and $\left\|\Delta_{L}^{H}\right\|=\mathscr{O}_{p}\left(k / n^{\frac{1}{2}}\right)$, so (3.4) holds if $k^{2} / q^{\frac{1}{2}} n^{\frac{1}{2}} \rightarrow 0$ as $n \rightarrow \infty$. If $v_{i}$ have constant variance $\sigma^{2}$, this can be estimated by $\check{\sigma}^{2}=\check{\sigma}_{n}^{2}=n^{-1}\left(y_{n}-X_{n} \check{\beta}\right)^{\prime}\left(y_{n}-X_{n} \check{\beta}\right)$ (where ${ }^{` c a n}$ be either ${ }^{\sim}$ or $\left.^{\wedge}\right)$ and it then follows that $\check{\sigma}^{2}-\sigma^{2}=\mathscr{O}_{p}(k / n)$ at worst.

Remark 3. In Example II, writing $R_{n}=A_{n}+B_{n}$ with $A_{n}=\left[G_{1 n} X_{n} \beta, \ldots, G_{p n} X_{n} \beta\right]$, $B_{n}=\left[G_{1 n} v, \ldots, G_{p n} v\right]$ and $G_{i n}=W_{i n} S_{n}^{-1}, i=1, \ldots, p$, we have $g_{n}=n^{-1}\left[R_{n}, X_{n}\right]^{\prime} v$, $M_{n}=\left[A_{n}, X_{n}\right], H_{n}=n^{-1}\left[R_{n}, X_{n}\right]^{\prime}\left[R_{n}, X_{n}\right]$ for OLS and $g_{n}=n^{-1}\left[R_{n}, X_{n}\right]^{\prime} \mathscr{P}\left(\left[Z_{n}, X_{n}\right]\right) v$, $M_{n}=\mathscr{P}\left(\left[Z_{n}, X_{n}\right]\right)\left[A_{n}, X_{n}\right], H_{n}=n^{-1}\left[R_{n}, X_{n}\right]^{\prime} \mathscr{P}\left(\left[Z_{n}, X_{n}\right]\right)\left[R_{n}, X_{n}\right]$ for IV. For PMLE, if $h \rightarrow \infty$ the same analysis as the OLS holds. Assumption 11 of Gupta and Robinson (2015) is designed to deal with the fact that the elements of $A_{n}$ can have divergent order of magnitude in increasing dimension SAR models, a natural consequence of their spatially dynamic nature. They further note that the elements of $A_{n}$ are uniformly $\mathscr{O}(k)$ if the elements of $X_{n}$ are taken to be uniformly bounded constants, implying $m=k$.

Under the conditions of Gupta and Robinson (2015), we have for OLS: $\left\|\Delta_{n^{-1} M^{\prime} v}^{g}\right\|=$ $\mathscr{O}_{p}\left(p^{\frac{1}{2}} / h\right),\left\|M_{n}^{\prime} v\right\|=\mathscr{O}_{p}\left(k(p n)^{\frac{1}{2}}\right),\left\|\Delta_{n^{-1} M^{\prime} M}^{H}\right\|=\mathscr{O}_{p}\left(\max \left\{p / h, p^{\frac{1}{2}} k(p+k)^{\frac{1}{2}} / n^{\frac{1}{2}}\right\}\right)$, and 
for IV: $\left\|\Delta_{n^{-1} M^{\prime} v}^{g}\right\|=\mathscr{O}_{p}\left(p^{\frac{1}{2}}(r+k) / n\right),\left\|\Delta_{n^{-1} M^{\prime} M}^{H}\right\|=\mathscr{O}_{p}\left(p^{\frac{1}{2}}(r+k)^{\frac{1}{2}} / n^{\frac{1}{2}}\right)$ and $\left\|M_{n}^{\prime} v\right\|=$ $\mathscr{O}_{p}\left((r+k)^{\frac{1}{2}} n^{\frac{1}{2}}\right)$. They also assume $\max \left\{p^{\frac{1}{2}} k, n^{\frac{1}{2}} p^{\frac{1}{2}} h^{-1}\right\}\left\|\Delta_{L}^{n^{-1} M^{\prime} M}\right\|=o_{p}(1)$ for OLS and $(r+k)^{\frac{1}{2}}\left\|\Delta_{L}^{n^{-1} M^{\prime} M}\right\|=o_{p}(1)$ for IV. So (3.4) holds under the following conditions as $n \rightarrow \infty:$

$$
\begin{array}{ll}
\text { OLS: } & \frac{1}{q^{\frac{1}{2}}}\left(\frac{p k n^{\frac{1}{2}}}{h}+\frac{p^{\frac{3}{2}} k^{3}(p+k)^{\frac{1}{2}}}{n^{\frac{1}{2}}}\right)+\frac{p}{h}+\frac{p^{\frac{1}{2}} k(p+k)^{\frac{1}{2}}}{n^{\frac{1}{2}}} \longrightarrow 0, \\
\mathrm{IV}: & \frac{p^{\frac{1}{2}}(r+k)^{\frac{3}{2}}}{q^{\frac{1}{2}} n^{\frac{1}{2}}}+\frac{p^{\frac{1}{2}}(r+k)^{\frac{1}{2}}}{n^{\frac{1}{2}}} \longrightarrow 0 .
\end{array}
$$

As far as relaxation to $\mathbb{E}\left(v_{i}^{2}\right)=\sigma^{2}$ is concerned, take

$$
\begin{aligned}
\hat{\sigma}_{S A R}^{2, O L S} & =n^{-1}\left(y_{n}-\left[R_{n}, X_{n}\right] \hat{\theta}_{S A R}^{O L S}\right)^{\prime}\left(y_{n}-\left[R_{n}, X_{n}\right] \hat{\theta}_{S A R}^{O L S}\right), \\
\hat{\sigma}_{S A R}^{2, I V} & =n^{-1}\left(y_{n}-\left[R_{n}, X_{n}\right] \hat{\theta}_{S A R}^{I V}\right)^{\prime}\left(y_{n}-\left[R_{n}, X_{n}\right] \hat{\theta}_{S A R}^{I V}\right) .
\end{aligned}
$$

By Theorems 3.2, 4.2 of Gupta and Robinson (2015), $\hat{\sigma}_{S A R}^{2, I V}-\sigma^{2}=\mathscr{O}_{p}((p+k)(r+k) / n)$ and $\hat{\sigma}_{S A R}^{2, O L S}-\sigma^{2}=\mathscr{O}_{p}\left(\max \left\{p k^{2}(p+k) / n, p / h\right\}\right)$

\section{Nonparametric regression specification testing with Property $\mathcal{C}$}

Sometimes it is not reasonable to assume a particular parametric form for the regression function, leading to consideration of

$$
y_{i}=d\left(x_{i}\right)+v_{i}, i=1, \ldots, n
$$

with $y_{i}$ observable, $x_{i}$ an $k \times 1$ vector of exogenous explanatory variables, $v_{i}$ is an independent and homoskedastic disturbance term and $d(\cdot)$ an unknown real-valued function on the support $\mathfrak{X}$ of $x_{i}$. On the other hand for multiple regression models such as (2.6), significance of the regression function can be tested by the null $H_{0}:\|\beta\|=0$ and various tests are available for functional form. We propose a specification test based on series estimation. The latter approximates $d(x)$ by $\alpha_{J}^{\prime} P_{J}(x)$, with $P_{J}(x)=\left(p_{1 J}(x), \ldots, p_{J J}(x)\right)^{\prime}$ and $\alpha_{J}=\left(\alpha_{1 J}, \ldots, \alpha_{J J}\right)^{\prime}$ being $J \times 1$ vectors of basis functions and unknown parameters respectively, and $J \rightarrow \infty$ slowly with $n$. Given an estimate $\hat{\alpha}_{J}$, we define a series estimate 
of $d(x)$ as

$$
\hat{d}(x)=\hat{\alpha}_{J}^{\prime} P_{J}(x)
$$

Andrews (1991) establishes a set of asymptotic normality results for more general settings including functionals of $d(\cdot)$, while uniform convergence rates of $\hat{d}(x)$ to $d(x)$ are derived by Newey (1997) in settings where the data are iid. Our interest is in testing null hypotheses on $d(x)$, which we will test by way of the 'approximate' null

$$
H_{0}^{a p p}:\left\|\alpha_{\mathfrak{t}(J)}-c_{\mathfrak{t}(J)}\right\|=0
$$

with $\alpha_{\mathfrak{t}(J)}$ a $\mathfrak{t}(J) \times 1$ subvector of $\alpha_{J}$ and $c_{\mathfrak{t}(J)}$ some known constant $\mathfrak{t}(J) \times 1$ vector, with $\mathfrak{t}(\cdot)$ an increasing integer valued (if the image is not an integer we choose the integer part of it) function. The alternative hypothesis will always be the negation of the null being tested.

This setup falls into the framework of Section 3.2. Indeed, $\hat{\alpha}_{J}$ can be formed by least squares regression of $y=\left(y_{1}, \ldots, y_{n}\right)^{\prime}$ on $P=P_{n}\left(x_{1}, \ldots, x_{n}\right)=\left[P_{J}\left(x_{1}\right), \ldots, P_{J}\left(x_{n}\right)\right]^{\prime}$, i.e. $\hat{\alpha}_{J}=\left(P^{\prime} P\right)^{-1} P^{\prime} y$, for sufficiently large $n$ if

$$
\varliminf_{n \rightarrow \infty} \underline{\eta}\left\{\mathbb{E}\left(P_{J}\left(x_{i}\right) P_{J}\left(x_{i}\right)^{\prime}\right)\right\}>0
$$

Thus take $\mathcal{Q}_{n}\left(\alpha_{J}\right)$ as in $(2.7)$ with $\psi(x)=x^{2} / 2$, implying that $g_{n}\left(\alpha_{J}\right)=n^{-1} P^{\prime}\left(P \alpha_{J}-y\right)$ and $H_{n}\left(\alpha_{J}\right)=n^{-1} P^{\prime} P$, which does not depend on $\alpha_{J}$. Assuming the existence of a $J \times 1$ constant vector $\alpha_{0 J}$ and scalar $\gamma>0$ such that

$$
\sup _{x \in \mathfrak{X}}\left|d(x)-\alpha_{0 J}^{\prime} P_{J}(x)\right|=\mathscr{O}_{p}\left(J^{-\gamma}\right), \text { as } J \rightarrow \infty,
$$

from Newey (1997), and substituting (4.1) gives $g_{n}=n^{-1} P^{\prime}\left(P \alpha_{0 J}-D(x)-v\right)$, with $D(x)=\left(d\left(x_{1}\right), \ldots, d\left(x_{n}\right)\right)^{\prime}$, whence

$$
\left\|\Delta_{-n^{-1} P^{\prime} v}^{g}\right\| \leq n^{-1}\|P\|\left\|P \alpha_{0 J}-D(x)\right\|=\mathscr{O}_{p}\left(J^{-\gamma}\right)
$$

As in the opening paragraph of the Appendix in Newey (1997), we can use (4.4) to normalize $P_{J}(x)$ so that Assumption 3 is satisfied with $m$ a constant sequence.

One case of interest is testing the significance of the nonparametric function, in which case take

$$
H_{0}^{\text {sig }}: d(x)=0
$$


$c_{J}=0($ so $\mathfrak{t}(J)=J)$ in (4.3). If the series functions are power series, we can use (4.3) as a more general specification test. To define these, let $\rho=\left(\rho_{1}, \ldots, \rho_{k}\right)^{\prime}$ be a multi-index with nonnegative entries, $z$ a $k \times 1$ real vector and denote $z^{\rho}=\prod_{j=1}^{k} z_{j}^{\rho_{j}}$. For a sequence $\{\rho(l)\}_{l=1}^{\infty}$ of distinct such vectors take $p_{j J}(x)=x^{\rho(j)}$. For instance, we may test for a linear regression

$$
H_{0}^{l i n}: d(x)=\alpha^{\prime} x
$$

by taking $c_{\mathfrak{t}(J)}$ to have zeros corresponding to indices $j$ for which $\rho(j)$ is not of the form whose only nonzero entry is unity, implying $\mathfrak{t}(J)=J-k$. In practice we must take $J>k$, which is satisfied in the theory as $k$ is fixed. $R$-th order polynomial regression can also be tested, taking $k=1$ for simplicity in notation:

$$
H_{0}^{\text {poly }}: d(x)=\sum_{i=1}^{R} \alpha_{i} x^{i},
$$

and $c_{\mathfrak{t}(J)}$ to have zeros corresponding to indices $j$ for which $\rho(j)$ has elements bigger than $R$, so $\mathfrak{t}(J)=J-R$. For general regression of order $R$ with $k$ explanatory variables the multinomial formula gives $\mathfrak{t}(J)=J-(k+R-1) ! /(k-1) ! R$ !. Other types of basis functions $p_{j J}(x)$ can yield specification tests against more functional parametric forms, indeed there is no dearth of options for the practitioner. To obtain tests of significance of a subset of regressors in $x_{i}$ as in Lavergne and Vuong (2000), we can test if all the $\alpha_{j J}$ corresponding to this subset are zero. Suppose $k=2$ and we are interested in testing if $x_{2 i}$ is a significant regressor. With a polynomial basis we would test if each $\alpha_{j J}$ that is a coefficient of a product involving $x_{2 i}$ is zero. Following a huge literature, parts of which have been cited in the introduction, we consider pointwise tests. Uniform theory such as those in Belloni, Chernozhukov, Chetverikov, and Kato (2015) is not explored in this paper, and would be much more relevant were we performing a test for nonparametric restrictions, such as shape restrictions implied by the Slutsky equation.

On the other hand the method proposed is not able to test against all general parametric alternatives. For example our approach cannot test if the regression function is a Box-Cox, i.e. $d(x)=\left(x^{\delta}-1\right) / \delta$ or $\operatorname{arcsinh}$ transformation, i.e. $d(x)=\sinh ^{-1}(\delta x) / \delta$, for some $\delta>0$, whereas the approach of Hong and White (1995) will work in this case.

An important alternative to (4.1) is the partly parametric model (see Robinson (1988), Fan and Li (1996)) where

$$
y_{i}=x_{1 i}^{\prime} \beta+d\left(x_{2 i}\right)+v_{i}, i=1, \ldots, n,
$$


with $x_{1 i}$ and $x_{2 i}$ subvectors of $x_{i}$. Our approach permits a simple way to test against this alternative. The practitioner only has to follow the method described in the paragraph above to test for linearity of the regressors in $x_{2 i}$. But we can offer something more if the linearity part of (4.10) is believed to hold. Our method gives a straightforward way to test for the specification of $d(\cdot)$, and this can be done in even more general cases where $x_{1 i}^{\prime} \beta$ is replaced by a parametric nonlinear function as in Andrews (1994).

Use of any of $\mathbf{L M}_{n}, \mathbf{W}_{n}$ or $\mathbf{L R}_{n}$ under $H_{0}^{a p p}$ provides a test for the question of interest with Property $\mathcal{C}$. This has some advantages compared to competing kernel based nonparametric specification tests. There is no need to choose a kernel (although $J$ needs to be chosen in practice), and the statistics are extremely simple to compute.

Proposition 4.1. Let $\left(y_{i}, x_{i}\right)$ be iid, $v_{i}$ satisfy the properties of $\epsilon_{i}$ in Assumption 2, (4.4) and (4.5) hold,

$$
\sup _{x \in \mathfrak{X}}\left\|P_{J}(x)\right\|=\mathscr{O}_{p}(\zeta(J)),
$$

for some function $\zeta(\cdot)$, and $J$ be chosen as function of $n$ satisfying (3.7) with $m$ constant, $s=J, q=\mathfrak{t}(J)$ and

$$
\frac{1}{J}+\frac{1}{\mathfrak{t}(J)}+\frac{\zeta^{2}(J) J}{n} \longrightarrow 0, \text { as } n \rightarrow \infty .
$$

Then $\mathbf{L M}_{n}, \mathbf{W}_{n}$ and $\mathbf{L} \mathbf{R}_{n}$ have Property $\mathcal{C}$, with $H_{0}^{\text {app }}$.

When $\mathfrak{X}$ is compact and connected and the support of $x_{i}$ has a pdf that is bounded away from zero, Newey (1997) derives $\zeta(J)=J$ for the power series basis and $\zeta(J)=J^{\frac{1}{2}}$ for a spline basis, the latter additionally assuming that $\mathfrak{X}$ is known. The value of $\gamma$ depends on features of $d(x)$ such as smoothness. For power series and spline bases, $\gamma=r / k$, where $r$ is the number of continuous derivatives of $d(x)$ on $\mathfrak{X}$, see Lorentz (1986). To satisfy (4.12) in the case of the power series basis described in the paragraph between (4.7) and (4.8) $J^{3} / n \rightarrow 0$ is sufficient.

An outstanding practical issue in series estimation is choice of $J$. Robinson (2005, 2010) discusses this, stating that asymptotic theory provides little guidance as it provides upper but not lower bounds. He points out that these upper bounds suggest very slow increase of $J$ and numerical experiments show that small integer choices of $J$ work well in practice.

The idea applies to more general models. Lee and Robinson (2016) consider series estimation in the presence of cross-sectional dependence, and we can easily accommodate this with (4.12) amended to the correct rates. Indeed, following that paper introduce the 
bivariate dependence measure

$$
\Upsilon_{n}=\frac{1}{n(n-1)} \sum_{\substack{i, j=1 \\ i \neq j}}^{n}\left\{\sup _{f \in F_{i j}}\left|\operatorname{cov}\left(f\left(x_{i}\right), f\left(x_{j}\right)\right)\right|\right\}, F_{i j}=\left\{f: \mathbb{E} f^{2}\left(x_{i}\right)=\mathbb{E} f^{2}\left(x_{j}\right)=1\right\}
$$

and replace (4.12) with

$$
\frac{1}{J}+\frac{1}{\mathfrak{t}(J)}+\frac{\zeta^{2}(J)\left(J+\Upsilon_{n}\right)}{n} \longrightarrow 0, \text { as } n \rightarrow \infty
$$

to obtain tests with Property $\mathcal{C}$. The latter is obtained from Assumption A.4 in Lee and Robinson (2016), noting that we have taken iid disturbances as opposed to their more general linear process specification. Indeed specification testing can undoubtedly extend to models such as the SAR in (2.11), where the linear regression component is replaced by a nonparametric function which is then estimated by series and tested for linearity, say, as in the previous paragraph. To the best of our knowledge the literature has not yet considered series estimation of the regression function in this setting, with kernel estimation seemingly the preferred tactic (see e.g. Su and Jin (2010), Jenish (2016)). The approach can also be used to test the specification of any of the fixed number of unknown varying coefficient functions $\vartheta_{j}(\cdot), j=1, \ldots, r_{1}$, in the model

$$
y_{i}=x_{1 i}^{\prime} \beta+x_{2 i}^{\prime} \vartheta\left(x_{3 i}\right)+v_{i}, i=1, \ldots, n,
$$

estimated using the series method by Ahmad, Leelahanon, and Li (2005), where $x_{2 i}$ and $x_{3 i}$ are $r_{1} \times 1$ and $r_{2} \times 1\left(r_{1}, r_{2}\right.$ fixed) vectors of exogenous explanatory variables respectively and $\vartheta\left(x_{3 i}\right)=\left(\vartheta_{1}\left(x_{3 i}\right), \ldots, \vartheta_{r_{1}}\left(x_{3 i}\right)\right)^{\prime}$.

\section{Error distribution specification in adaptive estima- tion}

In the adaptive estimation methodology of Newey (1988), which improves upon a treatment of Beran (1976), (2.6) is considered with unknown nonparametric density for a representative element $v_{i}$ of $v$. The aim is to obtain efficient estimates of $\beta$ by means of a Newton-type step that 'adapts' to the unknown error density using a series approximation, starting from an inefficient $n^{\frac{1}{2}}$-consistent initial estimate (such as OLS). It turns out 
that the object that must be nonparametrically estimated is not the density $f(t)$, but the score function $\varsigma(t)=-f_{t}(t) / f(t)$, where the $t$ subscript denotes partial derivative with respect to $t$. There are advantages to using series estimation for this, see Robinson (2010) p. 7 for details. To maintain simplicity we take $X_{n}$ to consist of uniformly bounded constants (as in Robinson (2010)). Let $\phi_{\ell}(t), \ell=1,2, \ldots$, be a sequence of smooth functions and let $J \geq 1$ be some user-chosen integer that increases slowly with $n$. Define $\phi^{(J)}(t)=\left(\phi_{1}(t), \ldots, \phi_{J}(t)\right)^{\prime}, \bar{\phi}^{(J)}(t)=\phi^{(J)}(t)-\mathbb{E}\left\{\phi^{(J)}\left(v_{i}\right)\right\}, \phi_{t}^{(J)}(t)=\left(\phi_{1 t}(t), \ldots, \phi_{J t}(t)\right)^{\prime}$. We approximate $\varsigma(t)$ by least squares projection on $\bar{\phi}^{(J)}(t)$. Denote the coefficients in this population projection by $a^{(J)}$. Then integration-by-parts leads to their identification by $a^{(J)}=\left[\mathbb{E}\left\{\bar{\phi}^{(J)}\left(v_{i}\right) \bar{\phi}^{(J)}\left(v_{i}\right)^{\prime}\right\}\right]^{-1} \mathbb{E}\left\{\phi_{v_{i}}^{(J)}\left(v_{i}\right)\right\}$. On other hand, we can write the sample equivalent

$$
\varsigma\left(t_{i}\right)=\Phi^{(J)}\left(t_{i}\right)^{\prime} a^{(J)}+u_{i}, \quad i=1, \ldots, n,
$$

for some zero mean, uncorrelated and homoskedastic (for ease of exposition we take their variance to be unity again) random variables $u_{i}$ that are independent of elements of $y_{n}$, and $\Phi^{(J)}\left(t_{i}\right)=\phi^{(J)}\left(t_{i}\right)-n^{-1} \sum_{j=1}^{n} \phi^{(J)}\left(t_{j}\right)$.

For an observable vector $e=\left(e_{1}, \ldots, e_{n}\right)^{\prime}$, approximate $a^{(J)}$ by $a^{\dagger(J)}(e)$, where for generic $t=\left(t_{1}, \ldots, t_{n}\right)^{\prime}, a^{\dagger(J)}(t)=W^{(J)}(t)^{-1} w^{(J)}(t)$ with $W^{(J)}(t)=n^{-1} \sum_{i=1}^{n} \Phi^{(J)}\left(t_{i}\right) \Phi^{(J)}\left(t_{i}\right)^{\prime}$, $w^{(J)}(t)=n^{-1} \sum_{i=1}^{n} \phi_{t_{i}}^{(J)}\left(t_{i}\right)$. The adaptive estimation literature then approximates $\varsigma\left(v_{i}\right)$ by $\Phi^{(J)}\left(e_{i}\right)^{\prime} a^{\dagger(J)}$ and inserts this in the Newton step for estimating $\beta$. Our interest in this paper lies in testing the specification of $\varsigma(\cdot)$, not the Newton step in which an estimate based on this specification is inserted. If there are indeed increasingly many nonzero elements in $a^{(J)}$ in (5.1), the adaptive estimation methodology will be efficient. On the other hand if there are only a finite number of nonzero elements in $a^{(J)}$, $\varsigma(t)$ will almost have a parametric form. By simply testing the significance of coefficients in the first stage of adaptive estimation for a range of $J$, the practitioner can employ a better specification and anticipate better performance of estimates. Thus we treat (5.1) like a linear regression model with increasingly many parameters and conduct tests of significance on increasingly large subvectors of $a^{(J)}$ using $\mathbf{L M}_{n}, \mathbf{W}_{n}$ or $\mathbf{L R}_{n}$. For example, if we take $\phi_{\ell}(t)=t^{\ell}$ and cannot reject the null hypothesis that all except the leading coefficient in (5.1) are zero then $\varsigma(t)$ is just the score function of a standard normal distribution. Writing $u=\left(u_{1}, \ldots, u_{n}\right)^{\prime}$, $\bar{W}^{(J)}(t)$ for the $n \times J$ matrix with typical row $\Phi^{(J)}\left(t_{i}\right)$ and taking $\psi(x)=x^{2} / 2$, we have $g_{n}=-n^{-1} \bar{W}^{\prime(J)} u$ (suppressing reference to the argument) and $H_{n}=n^{-1} \bar{W}^{\prime(J)} \bar{W}^{(J)}=$ $W^{(J)}$, so here $M_{n}=\bar{W}^{(J)}$. Assumption $\mathrm{A}^{*}$ in the proposition below defines the restrictions on $J$. It is quite technical and a repetition of conditions in Robinson (2005, 2010), so we 
state it in Appendix A. Simpler but stronger conditions restricting $J$ are given in Newey (1988).

Proposition 5.1. Suppose that we are testing the significance of a $\mathfrak{t}(J) \times 1$ subvector of $a^{(J)}$, where $\mathfrak{t}(J) \rightarrow \infty$ as $n \rightarrow \infty$, $u_{i}$ satisfy the properties of $\epsilon_{i}$ in Assumption 2, $X_{n}$ is formed of uniformly bounded constants and Assumption 3 holds, Assumption A* holds and $J$ is a function of $n$ satisfying (3.7) with $m$ constant, $s=J, q=\mathfrak{t}(J)$. Then $\mathbf{L M}_{n}, \mathbf{W}_{n}$ and $\mathbf{L R}_{n}$ have Property $\mathcal{C}$.

Testing nonparametric density specifications against parametric alternatives through simple inference on series coefficients is more widely applicable. Following Gallant and Nychka (1987), there is a very large literature on maximum likelihood estimation via series approximations to smooth unknown densities. A detailed treatment is beyond the scope of this paper, but to name one example Gurmu, Rilstone, and Stern (1999) consider semiparametric estimation of a count regression model based on a series expansion of the unknown density of unobserved heterogeneity. The approach of this paper is likely to be extendable to obtain density specification tests with Property $\mathcal{C}$ in this setting.

Consequences of misspecification in finite samples have effectively already been examined in a Monte Carlo study in Robinson (2010). In his design (cf. pg. 12 of that paper), adaptive estimates perform worse than the initial OLS for $J \geq 1$ when the true density of $v$ is standard normal, as expected. However there are no remedies in that paper to correct or test for the specification as we have proposed.

\section{Monte Carlo}

Finite sample implications of the theory were examined in a set of Monte Carlo experiments. The first of these analysed the performance of $\mathbf{L M}_{n}, \mathbf{W}_{n}$ and $\mathbf{L R}_{n}$ when $y_{n}$ was generated using (2.6), (2.9) and (2.11). The aim is to assess quality of inferences for small to moderate sample sizes and fairly large parameter spaces, and not to choose sample sizes so large and parameter spaces so small that the experiments become uninformative. Denoting true parameters with a zero subscript, the following designs were used to generate $y \equiv y_{n}$ in each of the 5000 replications:

(2.6) : $k=20,30,40, q=10,15,18, n=150,350,700, \beta_{0}=\iota_{k} / 2, X \sim U(0,1)$ but with first column of ones, $v \sim \mathcal{N}(0,1)$, 


$$
\begin{aligned}
(2.9): & k_{1}=k_{2}=2, q=T=5,10,15, N=50,100,200,\left(\alpha_{0}, \beta_{10}, \beta_{20}\right)=\left(\iota_{T}, \iota_{2} / 2, \iota_{2 T} / 2\right), \\
& X \sim U(0,5), v \sim \mathcal{N}(0,1), \\
(2.11): & k=2, q=p=8,16,32, m=12,48,96,\left(\lambda_{0}, \beta_{0}\right)=\left(U(0,1)_{p}, 1,1 / 2\right), X \sim U(0,5), \\
& W_{\text {in }}=\operatorname{diag}[0, \ldots, \underbrace{(m-1)^{-1}\left(\iota_{m} \iota_{m}^{\prime}-I_{m}\right)}_{i \text {-th diagonal block }}, \ldots, 0], v \sim \mathcal{N}(0,1) .
\end{aligned}
$$

The unusual notation in the choice of $\lambda_{0}$ indicates that the $\lambda_{0 i}$ were generated from $U(0,1)$ once at the start of the experiment (and then kept fixed for all combinations of $p$ and $m)$ to conform to a sufficient condition for the existence of a power series for $S_{n}^{-1}$ viz. $\left|\lambda_{0 i}\right|<1, i=1, \ldots, p$ (see Proposition 2.1 of Gupta and Robinson (2015)). They are: $0.81,0.91,0.13,0.91,0.63,0.1,0.28,0.55,0.96,0.96,0.16,0.97,0.96,0.49,0.8,0.14,0.42,0.92$ $0.79,0.96,0.66,0.04,0.85,0.93,0.68,0.76,0.74,0.39,0.66,0.17,0.71,0.03$.

Because $\mathbb{E}\left(W_{i n} y\right)=\mathbb{E}\left(W_{i n} S_{n}^{-1} X \beta_{0}\right)$ this allows instruments to be taken as linearly independent columns of $W_{i n}^{r} X, r \geq 1$. We maintain $r=1$ in our experiments, as is common in the SAR literature, for a total of $k p$ instruments apart from those in $X$, and analyse only IV estimates. The choice of $W_{i n}$ is commonly employed in Monte Carlo simulations using the SAR model and comes from Case $(1991,1992)$, who models an economy in which there are $p$ districts each with $m$ farmers who influence each farmer in their own district equally and are independent of farmers in other districts.

We first report empirical sizes and powers in Tables 6.1 and 6.2 for the following nulls:

$$
\begin{aligned}
& (2.6): H_{0}: \beta=\beta_{0}=\iota_{k} / 2, \quad H_{0}: \beta=0.4 \iota_{k}, \\
& (2.9): H_{0}: \alpha=\alpha_{0}=\iota_{T}, \quad H_{0}: \alpha=0.45 \iota_{T}, \\
& (2.11): H_{0}: \lambda=\lambda_{0}=U(0,1)_{p}, \quad H_{0}: \lambda_{i}=\lambda_{j}, i \neq j, i, j=1, \ldots, p .
\end{aligned}
$$

Note that the null for (2.11) is the one in (2.16). Tests of the type we construct are onesided because only positive increasing values occur asymptotically under the alternative hypothesis, so we employ one-sided critical values to compute the values in the tables. This was noted also by Hong and White (1995).

For (2.6) all three tests tend to be oversized compared to the nominal $5 \%$, but converging towards the latter as $n$ increases even though the improvement is not always monotonic, as seen by the behaviour of $\mathbf{L R}_{n}$ for $q=18$. oversizing worsens for all three tests as $k$ increases for given $n$ and also worsens for $\mathbf{W}_{n}$ and $\mathbf{L R}_{n}$ as $q$ increases for given $n$ and $k$ but improves for $\mathbf{L M}_{n}$, again with occasional exceptions to monotonicity. This reflects the fact 


\begin{tabular}{ccccccccccc}
$(2.6)$ & $n$ & \multicolumn{1}{c}{150} & \multicolumn{1}{c}{350} \\
\hline$k$ & $q$ & $\mathbf{W}_{n}$ & $\mathbf{L M}_{n}$ & $\mathbf{L R}_{n}$ & $\mathbf{W}_{n}$ & $\mathbf{L M}_{n}$ & $\mathbf{L R}_{n}$ & $\mathbf{W}_{n}$ & $\mathbf{L M}_{n}$ & $\mathbf{L R}_{n}$ \\
\hline 20 & 10 & 0.0820 & 0.0912 & 0.0834 & 0.0732 & 0.0756 & 0.0806 & 0.0650 & 0.0664 & 0.0724 \\
& 15 & 0.0886 & 0.0752 & 0.0908 & 0.0734 & 0.0684 & 0.0874 & 0.0672 & 0.0652 & 0.0856 \\
& 18 & 0.0872 & 0.0596 & 0.0952 & 0.0688 & 0.0574 & 0.0864 & 0.0674 & 0.0630 & 0.0916 \\
30 & 10 & 0.0736 & 0.1150 & 0.0752 & 0.0800 & 0.0932 & 0.0852 & 0.0684 & 0.0760 & 0.0800 \\
& 15 & 0.0794 & 0.1014 & 0.0792 & 0.0766 & 0.0848 & 0.0880 & 0.0690 & 0.0714 & 0.0866 \\
& 18 & 0.0796 & 0.0900 & 0.0840 & 0.0762 & 0.0788 & 0.0886 & 0.0690 & 0.0704 & 0.0946 \\
40 & 10 & 0.0836 & 0.1684 & 0.0792 & 0.0698 & 0.1026 & 0.0724 & 0.0660 & 0.0768 & 0.0756 \\
& 15 & 0.0894 & 0.1572 & 0.0856 & 0.0664 & 0.0892 & 0.0790 & 0.0662 & 0.0762 & 0.0862 \\
& 18 & 0.0914 & 0.1530 & 0.0892 & 0.0654 & 0.0876 & 0.0818 & 0.0672 & 0.0768 & 0.0902 \\
\hline$(2.9)$ & $N$ & & 50 & & & 100 & & & 200 & \\
\hline & $T$ & $\mathbf{W}_{n}$ & $\mathbf{L M}_{n}$ & $\mathbf{L R}_{n}$ & $\mathbf{W}_{n}$ & $\mathbf{L M}_{n}$ & $\mathbf{L R}_{n}$ & $\mathbf{W}_{n}$ & $\mathbf{L M}_{n}$ & $\mathbf{L R}_{n}$ \\
\hline & 5 & 0.1096 & 0.0810 & 0.0680 & 0.0900 & 0.0776 & 0.0704 & 0.0738 & 0.0654 & 0.0636 \\
& 10 & 0.1256 & 0.0838 & 0.0766 & 0.0894 & 0.0714 & 0.0732 & 0.0752 & 0.0682 & 0.0762 \\
& 15 & 0.1374 & 0.0858 & 0.0850 & 0.0928 & 0.0696 & 0.0886 & 0.0886 & 0.0776 & 0.0922 \\
\hline$(2.11)$ & $m$ & & 12 & & & 48 & & & 96 & \\
\hline & $p$ & $\mathbf{W}_{n}$ & $\mathbf{L M}_{n}$ & $\mathbf{L R}_{n}$ & $\mathbf{W}_{n}$ & $\mathbf{L M}_{n}$ & $\mathbf{L R}_{n}$ & $\mathbf{W}_{n}$ & $\mathbf{L M}_{n}$ & $\mathbf{L R}_{n}$ \\
\hline IV & 8 & 0.0914 & 0.2746 & 0.2036 & 0.0694 & 0.1058 & 0.0992 & 0.0704 & 0.0830 & 0.0856 \\
& 16 & 0.0900 & 0.2928 & 0.2226 & 0.0730 & 0.1058 & 0.1120 & 0.0660 & 0.0794 & 0.0958 \\
& 32 & 0.0884 & 0.4180 & 0.2838 & 0.0692 & 0.1230 & 0.1212 & 0.0650 & 0.0842 & 0.1006 \\
\hline
\end{tabular}

Table 6.1: Monte Carlo size for OLS for (2.6) and (2.9), IV for (2.11). Nominal size is 5\%.

that $\mathbf{L M}_{n}$ relies on estimation of the model under the null, which is more parsimonious, while the other two tests also require estimation of the unrestricted model. The powers for (2.6) displayed in the top panel of Table 6.2 indicate improvement again for larger samples in all tests. However power increases with $q$ (given $n, k$ ) for $\mathbf{W}_{n}$ and $\mathbf{L R}_{n}$ but decreases for $\mathbf{L M}_{n}$, with similar justification of this behaviour as provided for the sizes. Increase in $k$ (given $n$ ) doesn't seem to alter the behaviour of any test in a discernable pattern.

With (2.9) note that $n$ increases with $T$, which is also the rate of increase of the parameter space. There is oversizing with all three tests, but $\mathbf{L R}_{n}$ is the closest to the nominal 5\%. The oversizing worsens with increasing $T$ for given $N$ but not in each case, e.g. $\mathbf{L M}_{n}$ when $N=100$ actually improves with increasing $T$. The conclusion is that large $T$ can have serious consequences in inference on $T$ fixed-effect parameters even though it implies a larger $n=N T$. On the other hand, powers displayed in Table 6.2 for all three tests improve in all possible ways that $n$ can increase, viz. both $N$ and $T$ increase (diagonal), 


\begin{tabular}{ccccccccccc}
$(2.6)$ & $n$ & \multicolumn{1}{c}{150} & \multicolumn{1}{c}{350} \\
\hline$k$ & $q$ & $\mathbf{W}_{n}$ & $\mathbf{L M}_{n}$ & $\mathbf{L R}_{n}$ & $\mathbf{W}_{n}$ & $\mathbf{L M}_{n}$ & $\mathbf{L R}_{n}$ & $\mathbf{W}_{n}$ & $\mathbf{L M}_{n}$ & $\mathbf{L R}_{n}$ \\
\hline 20 & 10 & 0.1290 & 0.1404 & 0.1160 & 0.1952 & 0.2008 & 0.1890 & 0.3596 & 0.3628 & 0.3580 \\
& 15 & 0.1396 & 0.1200 & 0.1270 & 0.2304 & 0.2208 & 0.2268 & 0.4544 & 0.4456 & 0.4536 \\
& 18 & 0.1488 & 0.1088 & 0.1316 & 0.2564 & 0.2298 & 0.2470 & 0.4920 & 0.4814 & 0.4926 \\
30 & 10 & 0.1172 & 0.1684 & 0.0990 & 0.1962 & 0.2268 & 0.1936 & 0.3516 & 0.3682 & 0.3542 \\
& 15 & 0.1340 & 0.1686 & 0.1138 & 0.2270 & 0.2432 & 0.2164 & 0.4338 & 0.4436 & 0.4338 \\
& 18 & 0.1426 & 0.1560 & 0.1204 & 0.2456 & 0.2540 & 0.2382 & 0.4792 & 0.4822 & 0.4786 \\
40 & 10 & 0.1204 & 0.2298 & 0.1038 & 0.1954 & 0.2448 & 0.1928 & 0.3490 & 0.3812 & 0.3510 \\
& 15 & 0.1324 & 0.2334 & 0.1092 & 0.2258 & 0.2716 & 0.2176 & 0.4408 & 0.4682 & 0.4372 \\
& 18 & 0.1500 & 0.2288 & 0.1192 & 0.2426 & 0.2820 & 0.2290 & 0.4804 & 0.5048 & 0.4822 \\
\hline$(2.9)$ & $N$ & & 50 & & & 100 & & & 200 & \\
\hline & $T$ & $\mathbf{W}_{n}$ & $\mathbf{L M}_{n}$ & $\mathbf{L R}_{n}$ & $\mathbf{W}_{n}$ & $\mathbf{L M}_{n}$ & $\mathbf{L R}_{n}$ & $\mathbf{W}_{n}$ & $\mathbf{L M}_{n}$ & $\mathbf{L R}_{n}$ \\
\hline & 5 & 0.5478 & 0.4836 & 0.4568 & 0.8076 & 0.7798 & 0.7746 & 0.9854 & 0.9838 & 0.9852 \\
& 10 & 0.7490 & 0.6780 & 0.6520 & 0.9560 & 0.9464 & 0.9426 & 0.9996 & 0.9996 & 0.9998 \\
& 15 & 0.8620 & 0.7964 & 0.7696 & 0.9912 & 0.9888 & 0.9872 & 1.0000 & 1.0000 & 1.0000 \\
\hline$(2.11)$ & $m$ & & 12 & & & 48 & & & 96 & \\
\hline & $p$ & $\mathbf{W}_{n}$ & $\mathbf{L M}_{n}$ & $\mathbf{L R}_{n}$ & $\mathbf{W}_{n}$ & $\mathbf{L M}_{n}$ & $\mathbf{L R}_{n}$ & $\mathbf{W}_{n}$ & $\mathbf{L M}_{n}$ & $\mathbf{L R}_{n}$ \\
\hline IV & 8 & 0.1470 & 0.8198 & 1.0000 & 0.9314 & 0.9992 & 1.0000 & 1.0000 & 1.0000 & 1.0000 \\
& 16 & 0.1456 & 0.9654 & 1.0000 & 0.9966 & 1.0000 & 1.0000 & 1.0000 & 1.0000 & 1.0000 \\
& 32 & 0.1348 & 0.9986 & 1.0000 & 1.0000 & 1.0000 & 1.0000 & 1.0000 & 1.0000 & 1.0000 \\
\hline
\end{tabular}

Table 6.2: Monte Carlo power for OLS for (2.6) and (2.9), IV for (2.11).

only $N$ increases (horizontal), only $T$ increases (vertical). Unit power is attained by all three for the largest sample with $T=15, N=200$.

Finally, for (2.11) recall that $n$ increases with $p$, which is also the rate of increase of the parameter space. We find that $\mathbf{L} \mathbf{M}_{n}$ and $\mathbf{L} \mathbf{R}_{n}$ are unacceptably oversized for $m=12$, with $\mathbf{W}_{n}$ much better. However matters do improve for $\mathbf{L} \mathbf{M}_{n}$ and $\mathbf{L R}_{n}$ as $m$ increases, though the improvement is not necessarily better with both $m$ and $p$ increasing (so both parameter space and sample size increase) than with $m$ increasing for given $p$. In fact sizes are usually worse in the former case indicating that the gains for $\mathbf{L M}_{n}$ and $\mathbf{L} \mathbf{R}_{n}$ due to increased sample size are overpowered in this case by the burden of estimating extra parameters. On the other hand $\mathbf{W}_{n}$ showcases better performance and improves more as we proceed diagonally on the table as opposed to horizontally, reflecting a better response to increasing $n$ with both $m$ and $p$. The powers in Table 6.2 tell a somewhat different story, with $\mathbf{W}_{n}$ under-performing $\mathbf{L} \mathbf{M}_{n}$ and $\mathbf{L} \mathbf{R}_{n}$ substantially when $m=12$ but all tests 
giving excellent results for larger $m$. $\mathbf{L R}_{n}$ seems to be the clear winner here, always giving unit power, while the latter property is true for all three tests when $m=96$.

Our second set of experiments pertain to the specification testing procedure described in Section 4, using the power series basis described therein. In each of 5000 replications we generate $y_{i}, i=1, \ldots, n$ and $n=100,300,500$, using several data generating processes (DGPs):

$$
\begin{aligned}
D G P 1: y_{i} & =1+x_{1 i}+x_{2 i}+v_{i} \equiv x_{i}^{\prime} \beta_{0}+v_{i}, \\
D G P 2\left(\tau_{1}\right): y_{i} & =\exp \left\{\tau_{1}\left(x_{i}^{\prime} \beta_{0}\right)\right\}+v_{i}, \\
D G P 3\left(\tau_{2}\right): y_{i} & =\tau_{2} \log \left\{\log \left(x_{i}^{\prime} \beta_{0}\right)\right\}+v_{i}, \\
D G P 4\left(\tau_{3}\right): y_{i} & =1+\tau_{3}\left(\sin x_{1 i}+\cos x_{2 i}\right)+v_{i}, \\
D G P 5\left(\tau_{4}\right): y_{i} & =1+\tau_{4}\left(x_{1 i}^{-2}+x_{2 i}^{-\frac{1}{2}}\right)+v_{i}, \\
D G P 6: y_{i} & =1+0.8\left(x_{1 i} x_{2 i}\right)^{\frac{1}{2}}+0.1\left(x_{2 i} x_{3 i}\right)^{\frac{1}{2}}+v_{i}, \\
D G P 7: y_{i} & =1+\exp \left(0.1 x_{1 i} x_{2 i}\right)+x_{2 i} x_{3 i}+v_{i},
\end{aligned}
$$

where $x_{1 i}=\left(z_{i}+z_{1 i}\right) / 2, x_{2 i}=\left(z_{i}+z_{2 i}\right) / 2$ with $z_{i}, z_{1 i}, z_{2 i} \sim \operatorname{iid} U(0,5), v_{i} \sim$ iid $\mathcal{N}(0,1)$, $x_{3 i} \sim$ iid $\chi_{4}^{2}$ and $\tau_{i}, i=1,2,3,4$, some real numbers. Note that we have redefined $\beta_{0}=\iota_{3}$.

We experimented with $J=1,2,4$. (4.12) and the discussion in the paragraph below suggests an upper bound choice for $J$ of $\left[n^{\frac{1}{6}}\right]$, where we take $[x]$ to denote the integer part of $x$. This is is the kind of approach used by Hong and White (1995) when choosing $J$ in their simulations (they use closest integer, not integer part), but Robinson (2005, 2010) criticizes reliance on an upper bound for choice of $J$, stating that asymptotic theory provides little guidance. Our discussion of simulation results for DGP5 below give credence to this criticism. Our choices of $n$ imply $\left[n^{\frac{1}{6}}\right]=[2.15],[2.59],[2.82]=2,2,2$, but we choose to also report for $J=4$ to give a sharp illustration of the consequences of 'overfitting' the upper bound. For DGP5 and many cases of DGP6 we find that power improves, thus indicating that the upper bound may not always be the optimal choice.

When $J=1$ we are simply regressing $y$ on a constant, $x_{1}$ and $x_{2}$, and under the null we set both slope coefficients equal to zero. Thus the tests boil down to a test of significance of $x_{1}$ and $x_{2}$, and rejection probabilities are interpreted as power against an alternative that one of $x_{1}$ or $x_{2}$ is significant. For $J=2,4$ the null hypothesis sets all coefficients apart from those on $\left(1, x_{1}, x_{2}\right)$ to be zero. This null is true under $D G P 1$ so the rejection probabilities under this DGP are to be interpreted as sizes to be compared to the nominal 5\%, while 
under the other four DGPs the null is not true and the rejection probabilities are to be interpreted as power of a null hypothesis of linearity against these DGPs as alternatives.

The rejection probabilities are tabulated in Table 6.3. Under DGP1, the first row indicates unit power for all three tests and sample sizes. For $J \geq 2$, the next two rows display empirical sizes. All three tests are over-sized, but mostly acceptable for $n=500$ while even for $n=100,300$ they are not very far off with the best being $\mathbf{L M}_{n}$ with $J=2$. There is no clear winner overall between $J=2$ and $J=4$. Next we take $\tau_{1}=0.15,0.2$ in $D G P 2\left(\tau_{1}\right)$. Note that the expansion $\exp (x)=\sum_{j=0}^{\infty} x^{j} / j$ ! indicates that the smaller the absolute value of $\tau_{1}$ the closer $D G P 2\left(\tau_{1}\right)$ is to $D G P 1$. Thus tests against linearity are expected to have more power for larger $\tau_{1}$, which in fact should also lead to better power in tests of regressor significance. As discussed above our tests boil down to the latter when $J=1$, and in this case we see that we get unit power always. The power under a null of linearity is always better for $J=2$ than for $J=4$, although for both choices it increases with $n$. With $J=2$ and $\tau_{1}=0.2$, it starts at around $58 \%$ for all three tests when $n=100$ but improves to over $97 \%$ when $n=300$ and is around $99.9 \%$ for $n=500$. On the other hand the tests lose power quite dramatically for $\tau_{1}=0.15$, doing no better than $53.2 \%$, although they still improve with increasing $n$. Next we take $\tau_{2}=1.8,2$ for $D G P 3\left(\tau_{2}\right)$, finding rejection percentages when $J=1$ to be excellent for both cases with unit power achieved when $n=300,500$. For $n=100$ power is better for $\tau_{2}=2$, as we would anticipate. Power under the null of linearity follows much the same pattern, and like $D G P 2\left(\tau_{1}\right)$ power for $J=2$ dominates that for $J=4$. With both choices of $\tau_{2}$ power more than triples from $n=100$ to $n=500$, but from around $21 \%$ to around $71 \%$ when $\tau_{2}=1.8$ and from around $24 \%$ to around $79 \%$ when $\tau_{2}=2$. All three tests have very similar performance the larger $n$ becomes.

Next we study $D G P 4\left(\tau_{3}\right)$ with $\tau_{3}=0.2,0.5$. Power when the null imposes insignificance of the regressors becomes unity for $\tau_{3}=0.5, n \geq 300$, although it is over $95 \%$ even when $n=100$. With $\tau_{3}=0.2$, power is lower for $n=100$ but still very good for $n=500$. When the null is of linearity, $J=2$ has better power properties than $J=4$ for both values of $\tau_{3}$ but the power is much higher for the bigger value. An almost analogous analysis holds for $\operatorname{DGP5}\left(\tau_{4}\right)$, which we simulate with $\tau_{4}=0.3,0.1$. But, as mentioned earlier, there is one crucial difference: here $J=4$ dominates $J=2$, and in fact it does so for all tests, sample sizes and both values of $\tau_{4}$.

$D G P 6$ and $D G P 7$ have the distinctive feature of interaction terms in the nonlinear regression functions. For $D G P 7$ we record perfect performance of both regressor significance 
testing $(J=1)$ and rejections of linearity $(J=2,4)$. On the other hand we get worse performance with $D G P 6$, especially for the $\mathbf{W}_{n}$ when $n=100$. While matters improve with $n>100$ it is interesting to note that even in these cases $\mathbf{L R}_{n}$ and $\mathbf{L} \mathbf{M}_{n}$ perform much better. In all cases performance is better when $J=4$, as was the case with $D G P 5$, but the improvements can be quite dramatic.

\section{$7 \quad$ Empirical illustrations}

Data for both examples is available at https://www.economics.utoronto.ca/yatchew/.

\subsection{Scale economies in electricity distribution}

Yatchew (2000) considers (4.10) as a variant of the Cobb-Douglas model for the costs of distributing electricity with data on 81 municipal distributors in Ontario, Canada, during 1993. The interest lies in examining economies of scale in the number of customers. He takes $y=t c, x_{1}=(\text { wage, pcap, PUC, kwh, life, lf, kmwire })^{\prime}$ and $x_{2}=$ cust, where $t c$ is the log of total cost per customer, wage is the log wage rate, pcap is the log price of capital, $P U C$ is a dummy variable for public utility commissions that deliver additional services, $k w h$ is the log of kilowatt hour sales per customer, life is the log of remaining life of distribution assets, if is the log of the load factor (which measures capacity utilization relative to peak usage), kmwire is the log of kilometres of distribution wire per customer and cust is the log of the number of customers. Yatchew (2003) also fits a fully parametric specification with $d($ cust $)=\beta_{1}$ cust $+\beta_{2}$ cust $^{2}$. He uses a differencing procedure and his test (pg. 9) fails marginally to reject quadraticity, obtaining a test statistic of 1.5 to be compared to the $5 \%$ standard normal critical value of 1.645. However he later (pg. 77) employs a different specification test, also asymptotically standard normal, and finds that quadraticity is rejected with a statistic of 2.4. We will employ $\mathbf{W}_{n}$ to test for a quadratic specification, by fitting (4.10) using the series $\sum_{j=1}^{J} \alpha_{0 j} c_{u s t}{ }^{j}$ with $J=4,5$.

When $J=4, q=2$ and the null is $\alpha_{03}=\alpha_{04}=0$ while for $J=5, q=3$ and the null is $\alpha_{03}=\alpha_{04}=\alpha_{05}=0$. We get $\mathbf{W}_{n}=0.1,3.51$ for $J=4,5$ respectively. Compared to the one-sided $5 \%$ critical value of 1.645 for a standard normal distribution this results in a rejection of the null hypothesis of quadraticity when $J=5$. Because of the very small statistic when $J=4$ we conclude that including high order polynomial terms captures features of customer scale economies in electricity distribution that might otherwise be missed, thus providing evidence in favour of a semiparametric specification. 
Another basis is constructed using Laguerre polynomials, which have the the advantage of being orthogonal. These are given by the recursive relation

$$
L_{k+1}(x)=\frac{(2 k+1-x) L_{k}(x)-k L_{k-1}(x)}{k+1}, k \geq 1
$$

with $L_{0}(x)=1$ and $L_{1}(x)=1-x$. An estimation using $\sum_{j=1}^{J} \alpha_{0 j} L_{j}($ cust $)$ can be used to test for quadraticity by grouping polynomials of the same order and conducting significance tests on these as follows. Suppose $J=6$, and denote the coefficient on cust $^{j}, j=1, \ldots, 6$, by $\vartheta_{j}$. Then

$$
\begin{aligned}
\vartheta_{1} & =-\left(\alpha_{01}+2 \alpha_{02}+3 \alpha_{03}+4 \alpha_{04}+5 \alpha_{05}+6 \alpha_{06}\right) \\
\vartheta_{2} & =\frac{1}{2} \alpha_{02}+\frac{3}{2} \alpha_{03}+3 \alpha_{04}+5 \alpha_{05}+\frac{15}{2} \alpha_{06}, \\
\vartheta_{3} & =-\left(\frac{1}{6} \alpha_{03}+\frac{2}{3} \alpha_{04}+\frac{5}{3} \alpha_{05}+\frac{1}{3} \alpha_{06}\right) \\
\vartheta_{4} & =\frac{1}{24} \alpha_{04}+\frac{5}{24} \alpha_{05}+\frac{5}{8} \alpha_{06} \\
\vartheta_{5} & =-\left(\frac{1}{120} \alpha_{05}+\frac{1}{20} \alpha_{06}\right) \\
\vartheta_{6} & =\frac{1}{720} \alpha_{06} .
\end{aligned}
$$

It is clear that $\vartheta_{j}=0$ for all $j=2, \ldots, 6$, if and only if $\alpha_{0 j}=0$ for all $j=2, \ldots, 6$. Thus the analysis of the previous paragraph works also when using the Laguerre polynomial basis.

\subsection{Engel curve estimation: testing for linearity and equality}

Yatchew (2003) considers Engel curve estimation from South African household survey data. Two categories are considered: single individuals without children ('singles', 1,109 observations) and couples without children ('couples', 890 observations). For each group we estimate (4.1) with $y=f s$ and $x=\operatorname{texp}$ where $f s$ is the food share of total expenditure and texp is the log of total expenditure, using the series $\sum_{j=1}^{J} \alpha_{0 j} t e x p^{j}$ with $J=2,3,4$, plotting the results in Figure 7.1. One question of interest centres on whether the Engel curves are linear, see e.g. Lewbel (2008). To answer this question we test $\alpha_{02}=0$, $\alpha_{02}=\alpha_{03}=0, \alpha_{02}=\alpha_{03}=\alpha_{04}=0$ for $J=2,3,4$, respectively. Let $s$ and $c$ superscripts denote singles and couples respectively. We obtain $\mathbf{W}_{n}^{s}=10.47 ; \mathbf{W}_{n}^{c}=1.83$, for $J=2$, 
$\mathbf{W}_{n}^{s}=13.18 ; \mathbf{W}_{n}^{c}=17.07$, for $J=3$ and $\mathbf{W}_{n}^{s}=14.13 ; \mathbf{W}_{n}^{c}=13.98$, for $J=4$, indicating a rejection of the linear specification on comparison with a critical value of 1.645 . We thus opt for a nonparametric model with $J=4$, because taking $J>4$ leads to multicollinearity problems.
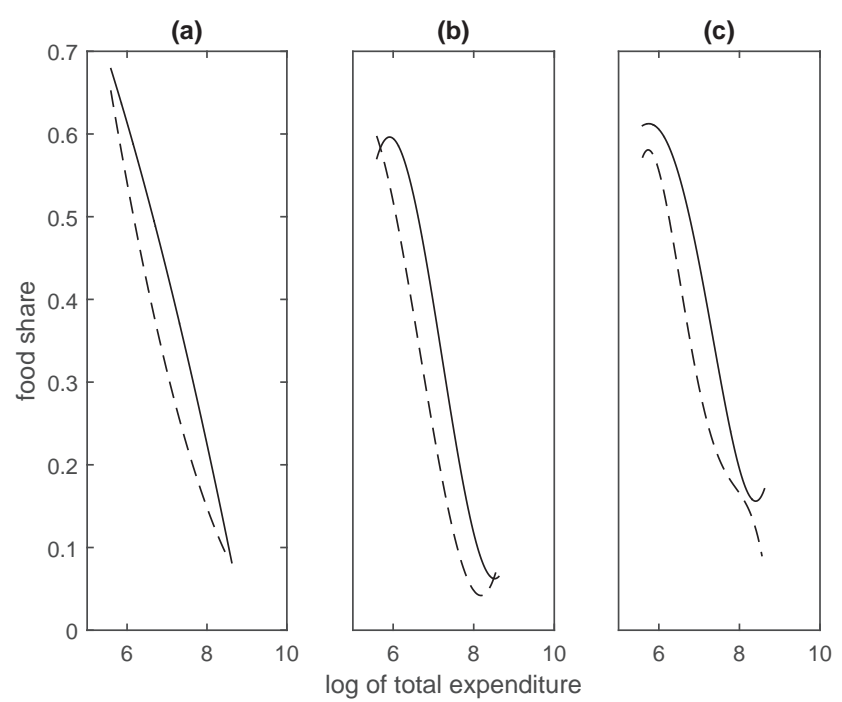

Figure 7.1: Fitted Engel curve for 'singles' (dashed line) and 'couples' (solid line), with $J=2(\mathrm{a}), J=3$ (b) and $J=4$ (c).

Once we have chosen our model (with $J=4$ ), an economic question of interest is: if the two Engel curves in Figure 7.1(c) were to be superimposed, would they coincide? It looks unlikely given the marked difference in shapes for high levels of texp. We answer this question by generating a dummy variable coup that takes the value 1 for couples and 0 otherwise, pooling the data for singles and couples, estimating the model

$$
f s=\alpha_{00}+\sum_{j=1}^{4} \alpha_{0 j} t \exp ^{j}+\sum_{j=1}^{4} \delta_{0 j}\left(\operatorname{coup} \times t \exp ^{j}\right)+v_{i}
$$

and testing $\delta_{01}=\delta_{02}=\delta_{03}=\delta_{04}=0$. This returns $\mathbf{W}_{n}=88.87$, implying a strong rejection of the null hypothesis. In contrast the test used in Yatchew (2003) (cf. p. 69) returned a statistic of 1.76 and therefore a rather weak rejection of the null of equality. 


\section{Conclusion and robustification to heteroskedastic- ity}

We have proposed nonparametric specification tests based on the trinity of testing principles for models in which objective function derivatives may be linear in iid disturbances. The test statistic sequences were shown to be asymptotically standard normal under the null hypothesis, consistent and possessing nontrivial power against local alternatives converging to the null at a prescribed rate. We now heuristically discuss heteroskedasticity robust versions of the tests. Suppose that the unit variance assumption on the $\epsilon_{i}$ is removed and instead $\operatorname{var}\left(\epsilon_{i}\right)=\sigma_{i}^{2}, i=1, \ldots, n$, where $\sigma_{i}^{-2} \leq C$. Note also that the existence of finite fourth moments guarantees that $\sigma_{i}^{2} \leq C$. Because so many of our cases of interest essentially involve only linear regression, we discuss heteroskedasticity robust versions of $\mathbf{L M}_{n}, \mathbf{L R}_{n}$ and $\mathbf{W}_{n}$ in (2.6). As for the homoskedastic case, these are obtained simply by standardizing usual heteroskedasticity robust versions of familiar statistics. Define $\hat{\epsilon}=\left(\hat{\epsilon}_{1}, \ldots, \hat{\epsilon}_{n}\right)^{\prime}, \tilde{\epsilon}=\left(\tilde{\epsilon}_{1}, \ldots, \tilde{\epsilon}_{n}\right)^{\prime}, \hat{\epsilon}_{i}=y_{i}-x_{i}^{\prime} \hat{\beta}, \tilde{\epsilon}_{i}=y_{i}-x_{i}^{\prime} \tilde{\beta}, \hat{\Omega}=\operatorname{diag}\left[\hat{\epsilon}_{1}^{2}, \ldots, \hat{\epsilon}_{n}^{2}\right]$,

$\tilde{\Omega}=\operatorname{diag}\left[\tilde{\epsilon}_{1}^{2}, \ldots, \tilde{\epsilon}_{n}^{2}\right], \hat{\mathcal{Q}}_{n}^{\mathcal{R}}=(y-X \hat{\beta})^{\prime} \hat{\Omega}^{-1}(y-X \hat{\beta}), \tilde{\mathcal{Q}}_{n}^{\mathcal{R}}=(y-X \tilde{\beta})^{\prime} \tilde{\Omega}^{-1}(y-X \tilde{\beta})$ and

$$
\begin{aligned}
\mathbf{L M}_{n}^{\mathcal{R}} & =\frac{\hat{\epsilon}^{\prime} \hat{\Omega}^{-1} X_{1}\left\{X_{1}^{\prime} \hat{\Omega}^{-1} X_{1}-X_{1}^{\prime} \hat{\Omega}^{-1} X_{2}\left(X_{2}^{\prime} \hat{\Omega}^{-1} X_{2}\right)^{-1} X_{2}^{\prime} \hat{\Omega}^{-1} X_{1}\right\}^{-1} X_{1}^{\prime} \hat{\Omega}^{-1} \hat{\epsilon}-q}{2^{\frac{1}{2}} q^{\frac{1}{2}}}, \\
\mathbf{L R}_{n}^{\mathcal{R}} & =\frac{2 n\left(\hat{\mathcal{Q}}_{n}^{\mathcal{R}}-\tilde{\mathcal{Q}}_{n}^{\mathcal{R}}\right)-q}{2^{\frac{1}{2}} q^{\frac{1}{2}}}, \\
\mathbf{W}_{n}^{\mathcal{R}} & =\frac{\tilde{\beta}_{1}^{\prime}\left\{X_{1}^{\prime} \tilde{\Omega}^{-1} X_{1}-X_{1}^{\prime} \tilde{\Omega}^{-1} X_{2}\left(X_{2}^{\prime} \tilde{\Omega}^{-1} X_{2}\right)^{-1} X_{2}^{\prime} \tilde{\Omega}^{-1} X_{1}\right\} \tilde{\beta}_{1}-q}{2^{\frac{1}{2}} q^{\frac{1}{2}}},
\end{aligned}
$$

where $X=\left[X_{1}, X_{2}\right]$ is a partition conformable to the dimension of the null. $\mathbf{L M}_{n}^{\mathcal{R}}, \mathbf{L R}_{n}^{\mathcal{R}}$ and $\mathbf{W}_{n}^{\mathcal{R}}$ can be shown to have Property $\mathcal{C}$ with similar techniques of proof as for $\mathbf{L} \mathbf{M}_{n}$, $\mathbf{L R}_{n}$ and $\mathbf{W}_{n}$. 


\begin{tabular}{|c|c|c|c|c|c|c|c|c|c|c|}
\hline \multirow[t]{2}{*}{$n$} & \multicolumn{4}{|c|}{100} & \multicolumn{3}{|c|}{300} & \multicolumn{3}{|c|}{500} \\
\hline & $J$ & $\mathbf{W}_{n}$ & $\mathbf{L M}_{n}$ & $\mathbf{L R}_{n}$ & $\mathbf{W}_{n}$ & $\mathbf{L M}_{n}$ & $\mathbf{L R}_{n}$ & $\mathbf{W}_{n}$ & $\mathbf{L M}_{n}$ & $\mathbf{L R}_{n}$ \\
\hline \multirow[t]{3}{*}{$D G P 1$} & 1 & 1.0000 & 1.0000 & 1.0000 & 1.0000 & 1.0000 & 1.0000 & 1.0000 & 1.0000 & 1.0000 \\
\hline & 2 & 0.0766 & 0.0754 & 0.0722 & 0.0758 & 0.0748 & 0.0712 & 0.0690 & 0.0686 & 0.0698 \\
\hline & 4 & 0.0882 & 0.0654 & 0.0792 & 0.0756 & 0.0694 & 0.0822 & 0.0690 & 0.0654 & 0.0858 \\
\hline \multirow[t]{3}{*}{$D G P 2(0.15)$} & 1 & 1.0000 & 1.0000 & 1.0000 & 1.0000 & 1.0000 & 1.0000 & 1.0000 & 1.0000 & 1.0000 \\
\hline & 2 & 0.1646 & 0.1620 & 0.1582 & 0.3670 & 0.3658 & 0.3666 & 0.5320 & 0.5310 & 0.5302 \\
\hline & 4 & 0.1288 & 0.0958 & 0.1148 & 0.2234 & 0.2108 & 0.2198 & 0.3178 & 0.3054 & 0.3176 \\
\hline \multirow[t]{3}{*}{$D G P 2(0.2)$} & 1 & 1.0000 & 1.0000 & 1.0000 & 1.0000 & 1.0000 & 1.0000 & 1.0000 & 1.0000 & 1.0000 \\
\hline & 2 & 0.5876 & 0.5812 & 0.5872 & 0.9752 & 0.9746 & 0.9756 & 0.9992 & 0.9992 & 0.9996 \\
\hline & 4 & 0.3914 & 0.3360 & 0.3734 & 0.8794 & 0.8686 & 0.8846 & 0.9890 & 0.9886 & 0.9890 \\
\hline \multirow[t]{3}{*}{$D G P 3(1.8)$} & 1 & 0.9092 & 0.9026 & 0.9142 & 1.0000 & 1.0000 & 1.0000 & 1.0000 & 1.0000 & 1.0000 \\
\hline & 2 & 0.2140 & 0.2090 & 0.2028 & 0.4818 & 0.4804 & 0.4832 & 0.7106 & 0.7098 & 0.7150 \\
\hline & 4 & 0.1784 & 0.1406 & 0.1618 & 0.3682 & 0.3528 & 0.3624 & 0.5752 & 0.5658 & 0.5724 \\
\hline \multirow[t]{3}{*}{$D G P 3(2)$} & 1 & 0.9534 & 0.9500 & 0.9576 & 1.0000 & 1.0000 & 1.0000 & 1.0000 & 1.0000 & 1.0000 \\
\hline & 2 & 0.2446 & 0.2390 & 0.2342 & 0.5642 & 0.5636 & 0.5692 & 0.7950 & 0.7944 & 0.7998 \\
\hline & 4 & 0.1996 & 0.1602 & 0.1840 & 0.4402 & 0.4204 & 0.4390 & 0.6758 & 0.6660 & 0.6746 \\
\hline \multirow[t]{3}{*}{$D G P 4(0.2)$} & 1 & 0.3396 & 0.3270 & 0.3342 & 0.7496 & 0.7476 & 0.7526 & 0.9260 & 0.9256 & 0.9292 \\
\hline & 2 & 0.1350 & 0.1312 & 0.1256 & 0.2444 & 0.2434 & 0.2446 & 0.3642 & 0.3628 & 0.3592 \\
\hline & 4 & 0.1156 & 0.0866 & 0.1082 & 0.1698 & 0.1582 & 0.1766 & 0.2538 & 0.2450 & 0.2542 \\
\hline \multirow[t]{3}{*}{$D G P 4(0.5)$} & 1 & 0.9550 & 0.9514 & 0.9624 & 1.0000 & 1.0000 & 1.0000 & 1.0000 & 1.0000 & 1.0000 \\
\hline & 2 & 0.4310 & 0.4254 & 0.4282 & 0.8832 & 0.8816 & 0.8880 & 0.9864 & 0.9864 & 0.9872 \\
\hline & 4 & 0.3166 & 0.2656 & 0.3006 & 0.7762 & 0.7642 & 0.7868 & 0.9556 & 0.9530 & 0.9594 \\
\hline \multirow[t]{3}{*}{$D G P 5(0.1)$} & 1 & 0.2212 & 0.2150 & 0.2564 & 0.4672 & 0.4648 & 0.5142 & 0.6222 & 0.6202 & 0.6600 \\
\hline & 2 & 0.2502 & 0.2480 & 0.2668 & 0.4984 & 0.4958 & 0.5186 & 0.6586 & 0.6580 & 0.6728 \\
\hline & 4 & 0.3036 & 0.2744 & 0.2982 & 0.6048 & 0.5954 & 0.6102 & 0.7700 & 0.7640 & 0.7744 \\
\hline \multirow[t]{3}{*}{$D G P 5(0.3)$} & 1 & 0.6040 & 0.5936 & 0.6446 & 0.9592 & 0.9586 & 0.9650 & 0.9966 & 0.9966 & 0.9976 \\
\hline & 2 & 0.5532 & 0.5500 & 0.5764 & 0.9166 & 0.9164 & 0.9240 & 0.9856 & 0.9854 & 0.9868 \\
\hline & 4 & 0.6016 & 0.5756 & 0.6050 & 0.9450 & 0.9414 & 0.9478 & 0.9956 & 0.9956 & 0.9958 \\
\hline \multirow[t]{3}{*}{$D G P 6$} & 1 & 0.4830 & 0.6184 & 0.6402 & 0.9110 & 0.9548 & 0.9582 & 0.9912 & 0.9966 & 0.9970 \\
\hline & 2 & 0.2106 & 0.5328 & 0.5162 & 0.4462 & 0.7780 & 0.7764 & 0.6532 & 0.8920 & 0.8936 \\
\hline & 4 & 0.2190 & 0.9448 & 0.8966 & 0.4280 & 0.9736 & 0.9710 & 0.6312 & 0.9918 & 0.9912 \\
\hline \multirow[t]{3}{*}{$D G P 7$} & 1 & 1.0000 & 1.0000 & 1.0000 & 1.0000 & 1.0000 & 1.0000 & 1.0000 & 1.0000 & 1.0000 \\
\hline & 2 & 1.0000 & 1.0000 & 1.0000 & 1.0000 & 1.0000 & 1.0000 & 1.0000 & 1.0000 & 1.0000 \\
\hline & 4 & 1.0000 & 1.0000 & 1.0000 & 1.0000 & 1.0000 & 1.0000 & 1.0000 & 1.0000 & 1.0000 \\
\hline
\end{tabular}

Table 6.3: Comparison of specification tests: Rejection probabilities 


\section{Appendices}

\section{A Proofs of theorems}

Proof of Theorem 3.1. We first carry out some preliminary development. Because $\theta_{10}=0$ under $H_{0}$, by the mean value (MVT) theorem we can write $\hat{g}_{1, n}=g_{1, n}+\bar{H}_{12, n} \Delta_{\theta_{20}}^{\hat{\theta}_{2}}$, $0=$ $\hat{g}_{2, n}=g_{2, n}+\bar{H}_{22, n} \Delta_{\theta_{20}}^{\hat{\theta}_{2}}$ with $\left\|\Delta_{\theta_{0}}^{\bar{\theta}}\right\| \leq\left\|\Delta_{\theta_{0}}^{\hat{\theta}}\right\|$, whence

$$
\hat{g}_{1, n}=\mathcal{Z}^{\bar{H}}\left[g_{1, n}^{\prime}, g_{2, n}^{\prime}\right]^{\prime}=\mathcal{Z}^{\bar{H}} g_{n}
$$

Note that the value of $\bar{\theta}$ may be different in each row of $\bar{H}_{12, n}$, this fact applying whenever the MVT is applied to a vector of values. Then (3.1) becomes

$$
\frac{n g_{n}^{\prime} \mathcal{H}_{n} g_{n}-q}{2^{\frac{1}{2}} q^{\frac{1}{2}}}
$$

where $\mathcal{H}_{n}=\mathcal{Z}^{\prime \bar{H}} \hat{H}_{n}^{11} \mathcal{Z}^{\bar{H}}$. Assumption 2 and Lemma B.5 imply that (A.2) is $\sum_{i=1}^{3} \Pi_{i, n}$ with

$$
\begin{aligned}
\Pi_{1, n} & =\frac{n \Delta_{n^{-1} M^{\prime} \epsilon}^{g} \mathcal{H}_{n} \Delta_{n^{-1} M^{\prime} \epsilon}^{g}}{2^{\frac{1}{2}} q^{\frac{1}{2}}}=\mathscr{O}_{p}\left(n\left\|\Delta_{n^{-1} M^{\prime} \epsilon}^{g}\right\|^{2} / q^{\frac{1}{2}}\right) \\
\Pi_{2, n} & =2 \frac{\epsilon^{\prime} M_{n} \mathcal{H}_{n} \Delta_{n^{-1} M^{\prime} \epsilon}^{g}}{2^{\frac{1}{2}} q^{\frac{1}{2}}}=\mathscr{O}_{p}\left(\left\|\Delta_{n^{-1} M^{\prime} \epsilon}^{g}\right\|\left\|M_{n}^{\prime} \epsilon\right\| / q^{\frac{1}{2}}\right) \\
\Pi_{3, n} & =\frac{n^{-1} \epsilon^{\prime} M_{n} \mathcal{H}_{n} M_{n}^{\prime} \epsilon-q}{2^{\frac{1}{2}} q^{\frac{1}{2}}} .
\end{aligned}
$$

(A.3), (A.4) are negligible by (3.4) so (A.2) is $\Pi_{3, n}+o_{p}(1)$. Denoting $\xi_{n}=\mathcal{Z}^{\prime \bar{H}} M_{n}^{\prime} \epsilon$ we have $\Pi_{3, n}=\left(n^{-1} \xi_{n}^{\prime} \hat{H}_{n}^{11} \xi_{n}-q\right) / 2^{\frac{1}{2}} q^{\frac{1}{2}}$. Note that by the proof of Lemma B.5, we have $\xi_{n}=\mathscr{O}_{p}\left(\left\|M_{n}^{\prime} \epsilon\right\|\right)$. We write $\Pi_{3, n}=\sum_{i=1}^{4} \Gamma_{3 i, n}$ with

$$
\begin{aligned}
\Gamma_{31, n} & =\frac{n^{-1} \xi_{n}^{\prime} \Delta_{H^{11}}^{\hat{H}^{11}} \xi_{n}}{2^{\frac{1}{2}} q^{\frac{1}{2}}}=\mathscr{O}_{p}\left(n^{-1}\left\|M_{n}^{\prime} \epsilon\right\|^{2}\left\|\Delta_{H}^{\hat{H}}\right\| / q^{\frac{1}{2}}\right), \\
\Gamma_{32, n} & =\frac{n^{-1} \xi_{n}^{\prime} \Delta_{n^{-1} M^{\prime} M^{11}} \xi_{n}}{2^{\frac{1}{2}} q^{\frac{1}{2}}}=\mathscr{O}_{p}\left(n^{-1}\left\|M_{n}^{\prime} \epsilon\right\|^{2}\left\|\Delta_{n^{-1} M^{\prime} M}^{H}\right\| / q^{\frac{1}{2}}\right), \\
\Gamma_{34, n} & =\frac{n^{-1} \xi_{n}^{\prime}\left(n^{-1} M_{n}^{\prime} M_{n}\right)^{11} \xi_{n}-q}{2^{\frac{1}{2}} q^{\frac{1}{2}}} .
\end{aligned}
$$


The bound for the $\Delta_{H^{11}}^{\hat{H}^{11}}$ term in (A.6) is justified by the following argument. First $\left\|\Delta_{H^{11}}^{\hat{H}^{11}}\right\| \leq\left\|H_{n}^{11}\right\|\left\|\hat{H}_{n}^{11}\right\|\left\|\Delta_{\left(H^{11}\right)^{-1}}^{\left(\hat{H}^{11}\right)^{-1}}\right\|$, with the first and second factors on the RHS $\mathscr{O}_{p}(1)$ by Lemma B.2. By the partitioned inverse formula $\left(\hat{H}_{n}^{11}\right)^{-1}=\hat{H}_{11, n}-\hat{H}_{12, n} \hat{H}_{22, n}^{-1} \hat{H}_{21, n}$ and $\left(H_{n}^{11}\right)^{-1}=H_{11, n}-H_{12, n} H_{22, n}^{-1} H_{21, n}$, implying that the last factor is bounded by $\left\|\Delta_{H_{11}}^{\hat{H}_{11}}\right\|+$ $\left\|\Delta_{H_{12} H_{22}^{-1} H_{21}}^{\hat{H}_{12} \hat{H}_{12}^{-1} \hat{H}_{21}}\right\|$. The first term in the last expression is bounded by $\left\|\Delta_{H}^{\hat{H}}\right\|$, while the second term is bounded by $\left\|\hat{H}_{12, n}\right\|\left\|\hat{H}_{21, n}\right\|\left\|H_{22, n}^{-1}\right\|\left\|\hat{H}_{22, n}^{-1}\right\|\left\|\Delta_{H_{22}}^{\hat{H}_{22}}\right\|+\left\|H_{22, n}^{-1}\right\|\left\|\hat{H}_{21, n}\right\|\left\|\Delta_{H_{12}}^{\hat{H}_{12}}\right\|+$ $\left\|H_{22, n}^{-1}\right\|\left\|H_{12, n}\right\|\left\|\Delta_{H_{21}}^{\hat{H}_{21}}\right\|$. By Lemmas B.2 and B.3, we conclude that $\left\|\hat{H}_{12, n}\right\|,\left\|\hat{H}_{21, n}\right\|$, $\left\|H_{12, n}\right\|,\left\|\hat{H}_{22, n}^{-1}\right\|$ and $\left\|H_{22, n}^{-1}\right\|$ are all $\mathscr{O}_{p}(1)$. Because $\left\|\Delta_{H_{22}}^{\hat{H}_{22}}\right\|,\left\|\Delta_{H_{12}}^{\hat{H}_{12}}\right\|$ and $\left\|\Delta_{H_{21}}^{\hat{H}_{21}}\right\|$ are all bounded by $\left\|\Delta_{H}^{\hat{H}}\right\|$ the bound in (A.6) now follows, and the bound in (A.7) is justified similarly. By (3.4), (A.6), (A.7) are negligible. Finally we show that

$$
\frac{n^{-1} \xi_{n}^{\prime}\left(n^{-1} M_{n}^{\prime} M_{n}\right)^{11} \xi_{n}}{2^{\frac{1}{2}} q^{\frac{1}{2}}}-\frac{n^{-1} \epsilon^{\prime} \mathcal{M}_{n} \epsilon}{2^{\frac{1}{2}} q^{\frac{1}{2}}}=o_{p}(1) .
$$

Adding and subtracting $n^{-1} \xi_{n}^{\prime}\left(n^{-1} M_{n}^{\prime} M_{n}\right)^{11} \mathcal{Z}^{n^{-1} M^{\prime} M} M_{n}^{\prime} \epsilon / q^{\frac{1}{2}}$ to the LHS and combining terms yields

$$
\begin{aligned}
& \frac{1}{2^{\frac{1}{2}} q^{\frac{1}{2}}} n^{-1} \epsilon^{\prime} M_{n}\left(\mathcal{Z}^{\bar{H}}+\mathcal{Z}^{n^{-1} M^{\prime} M}\right)^{\prime}\left(n^{-1} M_{n}^{\prime} M_{n}\right)^{11}\left[0, \Delta_{\bar{H}_{12} \bar{H}_{22}^{-1}}^{n^{-1} M^{\prime} M_{12}\left(n^{-1} M^{\prime} M_{22}\right)^{-1}}\right] M_{n}^{\prime} \epsilon \\
& \left\|\Delta_{\bar{H}_{12} \bar{H}_{22}^{-1}}^{n^{-1} M^{\prime} M_{12}\left(n^{-1} M^{\prime} M_{22}\right)^{-1}}\right\| \leq \sum_{i=1}^{4}\left\|\Upsilon_{i, n}\right\| \text { with } \Upsilon_{1, n}=\Delta_{H_{12}}^{\bar{H}_{12}} \bar{H}_{22, n}^{-1}, \Upsilon_{2, n}=\Delta_{n^{-1} M^{\prime} M_{12}}^{H_{12}} \bar{H}_{22, n}^{-1}, \\
& \Upsilon_{3, n}=M^{\prime} M_{12, n} \bar{H}_{22, n}^{-1} \Delta_{\bar{H}_{22}}^{H_{22}} H_{22, n}^{-1} \text { and } \Upsilon_{4, n}=M^{\prime} M_{12, n}\left(n^{-1} M_{n}^{\prime} M_{n}\right)_{22}^{-1} \Delta_{H_{22}}^{n^{-1} M^{\prime} M_{22}} H_{22, n}^{-1} \text {, so } \\
& \left\|\Upsilon_{1, n}\right\| \leq\left\|\Delta_{H_{12}}^{\bar{H}_{12}}\right\|\left\|\bar{H}_{22, n}^{-1}\right\|=\mathscr{O}_{p}\left(\left\|\Delta_{H}^{\bar{H}}\right\|\right), \\
& \left\|\Upsilon_{2, n}\right\| \leq\left\|\Delta_{n^{-1} M^{\prime} M_{12}}^{H_{12}}\right\|\left\|\bar{H}_{22, n}^{-1}\right\|=\mathscr{O}_{p}\left(\left\|\Delta_{n^{-1} M^{\prime} M}^{H}\right\|\right), \\
& \left\|\Upsilon_{3, n}\right\| \leq\left\|M^{\prime} M_{12, n}\right\|\left\|\bar{H}_{22, n}^{-1}\right\|\left\|H_{22, n}^{-1}\right\|\left\|\Delta_{H_{22}}^{\bar{H}_{22}}\right\|=\mathscr{O}_{p}\left(\left\|\Delta_{H}^{\bar{H}}\right\|\right), \\
& \left\|\Upsilon_{4, n}\right\| \leq\left\|M^{\prime} M_{12, n}\right\|\left\|H_{22, n}^{-1}\right\|\left\|\left(n^{-1} M_{n}^{\prime} M_{n}\right)_{22}^{-1}\right\|\left\|\Delta_{n^{-1} M^{\prime} M_{22}}^{H_{22}}\right\|=\mathscr{O}_{p}\left(\left\|\Delta_{n^{-1} M^{\prime} M}^{H}\right\|\right),
\end{aligned}
$$

by Lemmas B.2, B.3, B.6 and proof of Lemma B.5. Thus, by Lemma B.6, (A.10) is

$$
\mathscr{O}_{p}\left(n^{-1}\left\|M_{n}^{\prime} \epsilon\right\|^{2} \max \left\{\left\|\Delta_{H}^{\bar{H}}\right\|,\left\|\Delta_{n^{-1} M^{\prime} M}^{H}\right\|\right\} / q^{\frac{1}{2}}\right)=o_{p}(1),
$$


proving (A.9). The theorem is now proved.

Proof of Theorem 3.2. By the MVT, $0=\tilde{g}_{n}=g_{n}+\bar{H}_{n} \Delta_{\theta_{0}}^{\tilde{\theta}}$, with $\left\|\Delta_{\theta_{0}}^{\bar{\theta}}\right\| \leq\left\|\Delta_{\theta_{0}}^{\tilde{\theta}}\right\|$, so solving yields $\tilde{\theta}_{1}=-\left[\bar{H}_{n}^{11}, \bar{H}_{n}^{12}\right] g_{n}$. Thus, upon substituting in $(3.2), \mathbf{W}_{n}$ is

$$
\begin{aligned}
& \frac{n^{-1} \epsilon^{\prime} M_{n}\left[n^{-1} M_{n}^{\prime} M_{n}^{11}, n^{-1} M_{n}^{\prime} M_{n}^{12}\right]^{\prime}\left(n^{-1} M_{n}^{\prime} M_{n}^{11}\right)^{-1}\left[n^{-1} M_{n}^{\prime} M_{n}^{11}, n^{-1} M_{n}^{\prime} M_{n}^{12}\right] M_{n}^{\prime} \epsilon-q}{2^{\frac{1}{2}} q^{\frac{1}{2}}} \\
&+\mathscr{O}_{p}\left(\left[\left\|\Delta_{n^{-1} M^{\prime} \epsilon}^{g}\right\|\left\{n\left\|\Delta_{n^{-1} M^{\prime} \epsilon}^{g}\right\|+\left\|M_{n}^{\prime} \epsilon\right\|\right\}+n^{-1}\left\|M_{n}^{\prime} \epsilon\right\|^{2}\left\{\left\|\Delta_{H}^{\tilde{H}}\right\|+\left\|\Delta_{n^{-1} M^{\prime} M}^{H}\right\|\right\}\right] / q^{\frac{1}{2}}\right) .
\end{aligned}
$$

By (3.4) the second term above is negligible. The quadratic form in $M_{n}^{\prime} \epsilon$ in the first term is weighted by a matrix of rank $q$, which by the partitioned matrix inversion formula simplifies to $\left(n^{-1} M_{n}^{\prime} M_{n}\right)^{-1}-\Sigma^{n^{-1} M^{\prime} M}$, whence the claim follows. Now consider $\mathbf{L R}_{n}$. By the MVT we have

$$
\begin{aligned}
\mathbf{L R}_{n} & =\frac{n \Delta_{\tilde{\theta}}^{\prime \hat{\theta}} \bar{H}_{n} \Delta_{\tilde{\theta}}^{\hat{\theta}}-q}{2^{\frac{1}{2}} q^{\frac{1}{2}}}, \\
0 & =\tilde{g}_{1 n}=g_{1 n}+\bar{H}_{11, n} \tilde{\theta}_{1}+\bar{H}_{12, n} \Delta_{\theta_{20}}^{\tilde{\theta}_{2}}, \\
0 & =\tilde{g}_{2 n}=g_{2 n}+\bar{H}_{21, n} \tilde{\theta}_{1}+\bar{H}_{22, n} \Delta_{\theta_{20}}^{\tilde{\theta}_{2}}, \\
0 & =\hat{g}_{2 n}=g_{2 n}+\breve{H}_{22, n} \Delta_{\theta_{20}}^{\hat{\theta}_{2}},
\end{aligned}
$$

with $\left\|\Delta_{\theta_{0}}^{\bar{\theta}}\right\| \leq\left\|\Delta_{\theta_{0}}^{\tilde{\theta}}\right\|$ and $\left\|\Delta_{\theta_{0}}^{\breve{\theta}}\right\| \leq\left\|\Delta_{\theta_{0}}^{\hat{\theta}}\right\|$. Subtracting (A.18) from (A.17) gives $\bar{H}_{21, n} \tilde{\theta}_{1}+$ $\bar{H}_{22, n} \Delta_{\theta_{20}}^{\tilde{\theta}_{2}}=\breve{H}_{22, n} \Delta_{\theta_{20}}^{\hat{\theta}_{2}}$. Adding and subtracting $\bar{H}_{22, n} \hat{\theta}_{2}$ to the LHS gives $\bar{H}_{21, n} \tilde{\theta}_{1}+$ $\bar{H}_{22, n} \Delta_{\hat{\theta}_{2}}^{\tilde{\theta}_{2}}=\Delta_{\bar{H}_{22}}^{\breve{H}_{22}} \Delta_{\theta_{20}}^{\hat{\theta}_{2}}$, solving which we get $\Delta_{\hat{\theta}_{2}}^{\tilde{\theta}_{2}}=\left[\bar{H}_{22, n}^{-1} \bar{H}_{21, n}, \bar{H}_{22, n}^{-1} \Delta_{\bar{H}_{22}}^{\breve{H}_{22}}\right]\left(\tilde{\theta}_{1}^{\prime}, \Delta_{\theta_{20}}^{\prime \hat{\theta}_{2}}\right)^{\prime}$, implying

$$
\Delta_{\tilde{\theta}}^{\hat{\theta}}=\mathcal{Z}^{\prime \bar{H}} \tilde{\theta}_{1}+\left(0^{\prime},\left(\bar{H}_{22, n}^{-1} \Delta_{\bar{H}_{22}}^{\breve{H}_{22}} \Delta_{\theta_{20}}^{\hat{\theta}_{2}}\right)^{\prime}\right)^{\prime}
$$

Substituting (A.19) into (A.15), it follows by techniques similar to those used in earlier proofs that $\mathbf{L R}_{n}$ equals

$$
\frac{n \tilde{\theta}_{1}^{\prime} \mathcal{Z}^{n^{-1} M^{\prime} M}\left(n^{-1} M_{n}^{\prime} M_{n}\right) \mathcal{Z}^{n^{-1} M^{\prime} M} \tilde{\theta}_{1}-q}{2^{\frac{1}{2}} q^{\frac{1}{2}}}+o_{p}(1)=\frac{n \tilde{\theta}_{1}^{\prime}\left(n^{-1} M_{n}^{\prime} M_{n}^{11}\right)^{-1} \tilde{\theta}_{1}-q}{2^{\frac{1}{2}} q^{\frac{1}{2}}}+o_{p}(1),
$$

by (3.4) and the partitioned matrix inverse formula. The proof now follows that for $\mathbf{W}_{n}$.

Proof of Theorem 3.3. Denote by $\mathfrak{m}_{r t, n}$ the $(r, t)$-th element of $\mathcal{M}_{n}$. We seek to establish 
asymptotic normality of

$$
\frac{1}{2^{\frac{1}{2}} q^{\frac{1}{2}}} \sum_{r=1}^{n}\left(\epsilon_{r}^{2}-1\right) \mathfrak{m}_{r r, n}+\frac{2}{2^{\frac{1}{2}} q^{\frac{1}{2}}} \sum_{r=1}^{n} \epsilon_{r} \sum_{t<r} \epsilon_{t} \mathfrak{m}_{r t, n}=\sum_{r=1}^{n} z_{r n},
$$

say, with

$$
z_{r n}=\frac{1}{2^{\frac{1}{2}} q^{\frac{1}{2}}}\left(\epsilon_{r}^{2}-1\right) \mathfrak{m}_{r r, n}+\frac{2}{2^{\frac{1}{2}} q^{\frac{1}{2}}} \epsilon_{r} \sum_{t<r} \epsilon_{t} \mathfrak{m}_{r t, n} .
$$

We apply Theorem 2 of Scott (1973). Because the limit distribution is independent of the elements of $M_{n}$ we can prove the result conditional on these and, henceforth, all expectations will be conditional on elements of $M_{n}$. First note that by Lemma B.1, $\sum_{r=1}^{n} z_{r n}$ has mean zero and variance converging to 1 in probability. Writing $\mathbb{1}(\cdot)$ for indicator function, we need to prove

$$
\begin{aligned}
& \sum_{r=1}^{n} \mathbb{E}\left\{z_{r n}^{2} \mathbb{1}\left(\left|z_{r n}\right| \geq \epsilon\right)\right\} \stackrel{p}{\longrightarrow} 0, \text { for any } \epsilon>0, \\
& \sum_{r=1}^{n} \mathbb{E}\left(z_{r n}^{2} \mid \epsilon_{t}, t<r\right) \stackrel{p}{\longrightarrow} 1 .
\end{aligned}
$$

To show (A.22) we can check the sufficient Lyapunov condition

$$
\sum_{r=1}^{n} \mathbb{E}\left|z_{r n}\right|^{2+\frac{\chi}{2}} \stackrel{p}{\longrightarrow} 0
$$

Showing (A.24) boils down to proving that

$$
\begin{gathered}
\frac{1}{q^{1+\frac{\chi}{4}}} \sum_{r=1}^{n} \mathbb{E}\left|\epsilon_{r}^{2}-1\right|^{2+\frac{\chi}{2}}\left|\mathfrak{m}_{r r, n}\right|^{2+\frac{\chi}{2}} \stackrel{p}{\longrightarrow} 0, \\
\frac{1}{q^{1+\frac{\chi}{4}}} \sum_{r=1}^{n} \mathbb{E}\left|\epsilon_{r}\right|^{2+\frac{\chi}{2}} \mathbb{E}\left|\sum_{t<r} \epsilon_{t} \mathfrak{m}_{r t, n}\right|^{2+\frac{\chi}{2}} \stackrel{p}{\longrightarrow} 0
\end{gathered}
$$

By Assumption 2, (A.25) is bounded by a constant times

$$
\frac{n}{q^{1+\frac{\chi}{4}}} \max _{1 \leq r \leq n}\left|\mathfrak{m}_{r r, n}\right|^{2+\frac{\chi}{2}},
$$

while by the Jensen, Burkholder, $c_{r}$, von Bahr-Esseen, triangle and Hölder inequalities 
(A.26) is bounded by a constant times

$$
\begin{aligned}
& \frac{n}{q^{1+\frac{\chi}{4}}} \max _{1 \leq r \leq n} \mathbb{E}\left|\sum_{t<r} \epsilon_{t} \mathfrak{m}_{r t, n}\right|^{2+\frac{\chi}{2}} \leq \frac{C n}{q^{1+\frac{\chi}{4}}} \max _{1 \leq r \leq n}\left(\mathbb{E}\left|\sum_{t<r} \epsilon_{t} \mathfrak{m}_{r t, n}\right|^{4+\chi}\right)^{\frac{1}{2}} \\
\leq & \frac{C n}{q^{1+\frac{\chi}{4}}} \max _{1 \leq r \leq n}\left(\mathbb{E}\left|\sum_{t<r} \epsilon_{t}^{2} \mathfrak{m}_{r t, n}^{2}\right|^{2+\frac{\chi}{2}}\right)^{\frac{1}{2}} \leq \frac{C n}{q^{1+\frac{\chi}{4}}} \max _{1 \leq r \leq n}\left(\mathbb{E}\left|\sum_{t<r} \epsilon_{t}^{4} \mathfrak{m}_{r t, n}^{4}\right|^{1+\frac{\chi}{4}}\right)^{\frac{1}{2}} \\
\leq & \frac{C n}{q^{1+\frac{\chi}{4}}} \max _{1 \leq r \leq n}\left(\mathbb{E}\left|\sum_{t<r}\left(\epsilon_{t}^{4}-\mu_{4}\right) \mathfrak{m}_{r t, n}^{4}\right|^{1+\frac{\chi}{4}}+\left|\sum_{t<r} \mathfrak{m}_{r t, n}^{4}\right|^{1+\frac{\chi}{4}}\right)^{\frac{1}{2}} \\
\leq & \frac{C n}{q^{1+\frac{\chi}{4}}} \max _{1 \leq r \leq n}\left(\sum_{t<r} \mathbb{E}\left|\epsilon_{t}^{4}-\mu_{4}\right|^{1+\frac{\chi}{4}}\left|\mathfrak{m}_{r t, n}^{4}\right|^{1+\frac{\chi}{4}}+\left|\sum_{t<r} \mathfrak{m}_{r t, n}^{4}\right|^{1+\frac{\chi}{4}}\right)^{\frac{1}{2}} \\
\leq & \frac{C n}{q^{1+\frac{\chi}{4}}} \max _{1 \leq r \leq n}\left\{\left(\sum_{t<r} \mathfrak{m}_{r t, n}^{4}\right)^{1+\frac{\chi}{4}}\right\}^{\frac{1}{2}} \leq \frac{C n}{q^{1+\frac{\chi}{4}}} \max _{1 \leq r \leq n}\left(\sum_{t<r} \mathfrak{m}_{r t, n}^{2}\right)^{1+\frac{\chi}{4}} .
\end{aligned}
$$

For (A.27), note that $\mathfrak{m}_{r t, n}=n^{-1} m_{r n}^{\prime} \mathcal{Z}^{\prime n^{-1} M^{\prime} M}\left(n^{-1} M_{n}^{\prime} M_{n}\right)^{11} \mathcal{Z}^{n^{-1} M^{\prime} M} m_{t n}$ where $m_{r n}^{\prime}$ is the $r$-th row of $M_{n}$. Then

$$
\left|\mathfrak{m}_{r t, n}\right|=\mathscr{O}_{p}\left(n^{-1}\left\|m_{r n}\right\|\left\|m_{t n}\right\|\right)=\mathscr{O}_{p}\left(n^{-1} s m^{2}\right)
$$

by Lemma B.6 and Assumption 3. This implies that (A.27) is $\mathscr{O}_{p}\left(\left(s^{\frac{1}{2}} m\right)^{4+\chi} / n^{1+\frac{\chi}{2}} q^{1+\frac{\chi}{4}}\right)$, which is negligible by (3.7). For (A.28), $\sum_{t=1}^{n} \mathfrak{m}_{r t, n}^{2}$ equals $n^{-1}$ times

$$
\begin{aligned}
m_{r n}^{\prime} \mathcal{Z}^{n^{-1} M^{\prime} M} & \left(n^{-1} M_{n}^{\prime} M_{n}\right)^{11} \mathcal{Z}^{n^{-1} M^{\prime} M}\left(n^{-1} M_{n}^{\prime} M_{n}\right) \mathcal{Z}^{n^{-1} M^{\prime} M}\left(n^{-1} M_{n}^{\prime} M_{n}\right)^{11} \mathcal{Z}^{n^{-1} M^{\prime} M} m_{r n} \\
& \leq\left\|m_{r n}\right\|^{2}\left\|\mathcal{Z}^{\prime n^{-1} M^{\prime} M}\left(n^{-1} M_{n}^{\prime} M_{n}\right)^{11} \mathcal{Z}^{n^{-1} M^{\prime} M}\right\|^{2}\left\|n^{-1} M_{n}^{\prime} M_{n}\right\|=\mathscr{O}_{p}\left(s m^{2}\right)
\end{aligned}
$$

Then (A.28) is $\mathscr{O}_{p}\left(\left(s^{\frac{1}{2}} m\right)^{2+\frac{\chi}{2}} / n^{\frac{\chi}{4}} q^{1+\frac{\chi}{4}}\right)$, which is negligible by (3.7).

To show (A.23) note that by Lemma B.1 $\sum_{r=1}^{n} \mathbb{E}\left(z_{r n}^{2} \mid \epsilon_{t}, t<r\right)-1$ equals

$$
\frac{4}{2 q} \sum_{r=1}^{n} \sum_{\substack{t, u<r \\ t \neq u}} \epsilon_{t} \epsilon_{u} \mathfrak{m}_{r t, n} \mathfrak{m}_{r u, n}+\frac{1}{2 q} \sum_{r=1}^{n} \sum_{t<r}\left(\epsilon_{t}^{2}-1\right) \mathfrak{m}_{r t, n}^{2}+\frac{4}{2 q} \sum_{r=1}^{n} \mu_{3} \mathfrak{m}_{r r, n} \sum_{t<r} \epsilon_{t} \mathfrak{m}_{r t, n}
$$


Also, because $\mathcal{M}_{n}$ is idempotent and symmetric, we have, for $r, t=1, \ldots, n$,

$$
\mathfrak{m}_{r t, n}=\sum_{u=1}^{n} \mathfrak{m}_{r u, n} \mathfrak{m}_{u t, n}=\sum_{u=1}^{n} \mathfrak{m}_{r u, n} \mathfrak{m}_{t u, n}
$$

i.e. $\mathcal{M}_{n}$ and $\mathcal{M}_{n}^{2}$ have the same elements. In particular this indicates that

$$
\operatorname{tr}\left(\mathcal{M}_{n}^{2}\right)=\sum_{r} \sum_{u} \mathfrak{m}_{r u, n} \mathfrak{m}_{r u, n}=\sum_{r, u} \mathfrak{m}_{r u, n}^{2}
$$

The first term in (A.30) has zero mean and (conditional) variance bounded by $1 / q^{2}$ times

$$
\begin{aligned}
C \sum_{\substack{v, r, t, u \\
t, u<r, v}} \mathfrak{m}_{r t, n} \mathfrak{m}_{r u, n} \mathfrak{m}_{v t, n} \mathfrak{m}_{v u, n} & =C \sum_{\substack{t, u \\
t, u<r, v}} \sum_{r} \mathfrak{m}_{r u, n} \mathfrak{m}_{r t, n} \sum_{v} \mathfrak{m}_{v u, n} \mathfrak{m}_{v t, n} \\
& =C \sum_{\substack{t, u \\
t, u<r, v}} \mathfrak{m}_{t u, n}^{2} \leq C \sum_{t, u} \mathfrak{m}_{t u, n}^{2}=C q
\end{aligned}
$$

using (A.31), while the last equality follows because (A.32) and the idempotency of $\mathcal{M}_{n}$ imply that $\sum_{r, t} \mathfrak{m}_{r t, n}^{2}=\operatorname{tr}\left(\mathcal{M}_{n}^{2}\right)=\operatorname{tr}\left(\mathcal{M}_{n}\right)=q$, because $\mathcal{M}_{n}$ has rank $q$. Thus the first term in (A.30) is negligible because $q \rightarrow \infty$ as $n \rightarrow \infty$. The second term in (A.30) also has zero mean. By (A.29), (A.31) and (A.32), its variance is bounded by

$$
\begin{aligned}
C q^{-2} \sum_{r, v} \sum_{t<r, v} \mathfrak{m}_{r t, n}^{2} \mathfrak{m}_{v t, n}^{2} & \leq C q^{-2} \sum_{r, v, t} \mathfrak{m}_{r t, n}^{2} \mathfrak{m}_{v t, n}^{2} \\
& =C q^{-2} \sum_{r, t} \mathfrak{m}_{r t, n}^{2} \sum_{v} \mathfrak{m}_{v t, n}^{2}=C q^{-2} \sum_{r, t} \mathfrak{m}_{r t, n}^{2} \mathfrak{m}_{t t, n} \\
& \leq C q^{-2} \max _{t}\left|\mathfrak{m}_{t t, n}\right| \sum_{r, t} \mathfrak{m}_{r t, n}^{2}=\mathscr{O}_{p}\left(q^{-1} n^{-1} s m^{2}\right)
\end{aligned}
$$

which is again negligible under (3.7). Finally the third term in (A.30) has mean zero and variance bounded by $q^{-2} \max _{t} \mathfrak{m}_{t t, n}^{2} \sum_{r, t} \mathfrak{m}_{r t, n}^{2}=\mathscr{O}_{p}\left(q^{-1} n^{-2} s^{2} m^{4}\right)$, which is negligible.

Proof of Theorem 3.4. First note that $n^{-1} M_{n}\left(\left(n^{-1} M_{n}^{\prime} M_{n}\right)^{-1}-\Sigma^{n^{-1} M^{\prime} M}\right) M_{n}^{\prime}$ is symmetric and has rank $q$, and when multiplied by itself gives

$$
n^{-1} M_{n}\left\{\left(n^{-1} M_{n}^{\prime} M_{n}\right)^{-1}+n^{-1} \Sigma^{n^{-1} M^{\prime} M} M_{n}^{\prime} M_{n} \Sigma^{n^{-1} M^{\prime} M}-2 \Sigma^{n^{-1} M^{\prime} M}\right\} M_{n}^{\prime} .
$$

Now partitioning $n^{-1} M_{n}^{\prime} M_{n}$ yields $n^{-1} \Sigma^{n^{-1} M^{\prime} M} M_{n}^{\prime} M_{n} \Sigma^{n^{-1} M^{\prime} M}=\Sigma^{n^{-1} M^{\prime} M}$, so that $\mathbf{W}_{n}$ 
and $\mathbf{L R}_{n}$ are quadratic forms in $\epsilon$ weighted by a symmetric, idempotent matrix with rank $q$. The proof now follows that of Theorem 3.3 and the details are omitted.

Proof of Theorem 3.5. (i): $\mathcal{C} .1$ follows from Theorems 3.1 and 3.3. For $\mathcal{C} .2, n \hat{g}_{1, n}^{\prime} \hat{H}_{n}^{11} \hat{g}_{1, n}=$ $n g_{n}^{\prime 0} \mathcal{H}_{n} g_{n}^{0}$ by (A.2), where the 0 superscript denotes evaluation at $\left(0^{\prime}, \theta_{20}^{\prime}\right)^{\prime}$, which is no longer the true parameter value under $H_{1}$. Another application of the MVT yields

$$
g_{n}^{0}=g_{n}-\frac{\partial}{\partial \theta_{1}^{\prime}} g\left(\left(\stackrel{\circ}{\theta}_{1}^{\prime}, \theta_{20}^{\prime}\right)^{\prime}\right) \theta_{10}=g_{n}-\tau_{n}^{\stackrel{\circ}{H}}
$$

say, where $\left\|\stackrel{\circ}{\theta}_{1}\right\| \leq\left\|\theta_{10}\right\|$. Thus

$$
\frac{n g_{n}^{\prime 0} \mathcal{H}_{n} g_{n}^{0}-q}{2^{\frac{1}{2}} q^{\frac{1}{2}}}=\frac{n g_{n}^{\prime} \mathcal{H}_{n} g_{n}-q}{2^{\frac{1}{2}} q^{\frac{1}{2}}}-2 \frac{n \tau_{n}^{\prime H} \mathcal{H}_{n} g_{n}}{2^{\frac{1}{2}} q^{\frac{1}{2}}}+\frac{n \tau_{n}^{\prime H} \mathcal{H}_{n} \tau_{n}^{\prime \stackrel{\circ}{H}}}{2^{\frac{1}{2}} q^{\frac{1}{2}}}
$$

The first term converges in distribution to a standard normal variate, as in Theorem 3.3, and is therefore $\mathscr{O}_{p}(1)$. Because at least one element of $\theta_{10}$ is nonzero, the third term on the RHS of (A.36) is readily seen by Assumption 2 and Lemma B.5 to be at least $\mathscr{O}_{p}\left(n / q^{\frac{1}{2}}\right)$, and evidently dominates the second term. Thus the LHS of (A.36) diverges at $n / q^{\frac{1}{2}}$ rate, and therefore so does $\mathbf{L M}_{n}$, by Theorem 3.1. Hence, for all $\varepsilon>0, P\left(\left|\mathbf{L M}_{n}^{-1}\right| \leq \varepsilon q^{\frac{1}{2}} / n\right) \rightarrow$ 1 as $n \rightarrow \infty$, implying that, for all $\varrho>0$,

$$
P\left(\left|\mathbf{L M}_{n}\right|>\varrho\right) \rightarrow 1, \text { as } n \rightarrow \infty
$$

because $q^{\frac{1}{2}}=o(n)$. Denote $\stackrel{\circ}{\theta}=\left(\stackrel{\circ}{\theta}_{1}^{\prime}, \theta_{20}^{\prime}\right)^{\prime}$. To show $\mathcal{C} .3$ first note that under the sequence of local alternatives $H_{\ell 1}, \tau_{n}^{\stackrel{\circ}{H}}=\left[\stackrel{\circ}{H}_{11, n}, \stackrel{\circ}{H}_{12, n}\right]^{\prime} \theta_{\ell, 10}$. As $\left\|\theta_{\ell, 10}\right\| \rightarrow 0,\left\|\stackrel{\circ}{1}_{1}\right\| \rightarrow 0$ also and so $\left\|\Delta_{\theta_{0}}^{\stackrel{\ominus}{\theta}}\right\| \rightarrow 0$ under $H_{\ell 1}$. Thus by Assumption 2 and (3.7), and because $\left\|\theta_{\ell, 10}\right\|^{2}=\mathscr{O}\left(q^{\frac{1}{2}} / n\right)$ we can use (A.36) to write

$$
\begin{aligned}
\frac{n \tau_{n}^{\prime \stackrel{\circ}{H}} \mathcal{H}_{n} \tau_{n}^{\stackrel{\circ}{H}}}{2^{\frac{1}{2}} q^{\frac{1}{2}}}= & \mathscr{O}_{p}\left(\max \left\{\left\|\Delta_{H}^{\stackrel{\circ}{H}}\right\|,\left\|\Delta_{n^{-1} M^{\prime} M}^{H}\right\|,\left\|\Delta_{L}^{n^{-1} M^{\prime} M}\right\|\right\}\right)+\frac{n \tau_{n}^{\prime L} \mathcal{L}_{n} \tau_{n}^{L}}{2^{\frac{1}{2}} q^{\frac{1}{2}}} \\
\frac{n \tau_{n}^{\prime \prime} \mathcal{H}_{n} g_{n}}{2^{\frac{1}{2}} q^{\frac{1}{2}}}= & \mathscr{O}_{p}\left(n^{\frac{1}{2}} q^{-\frac{1}{4}}\left\|\Delta_{n^{-1} M^{\prime} \epsilon}^{g}\right\|\right)+\mathscr{O}_{p}\left(n ^ { - \frac { 1 } { 2 } } q ^ { - \frac { 1 } { 4 } } \| M _ { n } ^ { \prime } \epsilon \| \operatorname { m a x } \left\{\left\|\Delta_{H}^{\stackrel{\circ}{H}}\right\|,\right.\right. \\
& \left.\left.\left\|\Delta_{n^{-1} M^{\prime} M}^{H}\right\|,\left\|\Delta_{L}^{n^{-1} M^{\prime} M}\right\|\right\}\right)+\frac{\tau_{n}^{\prime L} \mathcal{L}_{n} M_{n}^{\prime} \epsilon}{2^{\frac{1}{2}} q^{\frac{1}{2}}}
\end{aligned}
$$


with $\mathcal{L}_{n}=\mathcal{Z}^{\prime L} L_{n}^{11} \mathcal{Z}^{L}$. Everything apart from the last terms on the RHSs of (A.38) and (A.39) are negligible by (3.7). Choosing $\Gamma_{n}=\left[L_{11, n}, L_{12, n}\right] \mathcal{L}_{n}\left[L_{11, n}, L_{12, n}\right]^{\prime}$ in $H_{\ell 1}$ the second term on the RHS of (A.38) equals $2^{-\frac{1}{2}}$, so that the LHS converges in probability to $2^{-\frac{1}{2}}$. The last term on the RHS of (A.39) has zero mean and conditional variance bounded by a constant times

$$
\begin{array}{r}
\delta_{n}^{\prime}\left[L_{11, n}, L_{12, n}\right] \mathcal{L}_{n}\left(n^{-1} M_{n}^{\prime} M_{n}\right) \mathcal{L}_{n}\left[L_{11, n}, L_{12, n}\right]^{\prime} \delta_{n} / q^{\frac{1}{2}} \delta_{n}^{\prime} \Gamma_{n} \delta_{n}=\delta_{n}^{\prime}\left[L_{11, n}, L_{12, n}\right] \mathcal{L}_{n} \times \\
\Delta_{L}^{n^{-1} M^{\prime} M} \mathcal{L}_{n}\left[L_{11, n}, L_{12, n}\right]^{\prime} \delta_{n} / q^{\frac{1}{2}} \delta_{n}^{\prime} \Gamma_{n} \delta_{n}+1 / q^{\frac{1}{2}}
\end{array}
$$

whose first term is $\mathscr{O}_{p}\left(\left\|\Delta_{L}^{n^{-1} M^{\prime} M}\right\| / q^{\frac{1}{2}}\right)=o_{p}(1)$, by Assumption 3, using the techniques of Lemma B.5 to conclude that norms of blocks of $L_{n}$ are $\mathscr{O}(1)$ and consequently so is $\mathcal{L}_{n}$. The simplification of the second term results from $\mathcal{L}_{n} L_{n} \mathcal{L}_{n}=\mathcal{L}_{n}$, because $\mathcal{Z}^{\prime L} L_{n} \mathcal{Z}^{L}=\left(L_{n}^{11}\right)^{-1}$ by the partitioned inverse formula. Thus the LHS of (A.39) is negligible, proving the result.

Part $(i i)$ is proved in an identical fashion and we omit the details, noting only that we take $\Gamma_{n}=\left[L_{11, n}, L_{12, n}\right]\left(L_{n}^{-1}-\Sigma^{L}\right)\left[L_{11, n}, L_{12, n}\right]^{\prime}$, which equals $\left[L_{11, n}, L_{12, n}\right] \mathcal{L}_{n}\left[L_{11, n}, L_{12, n}\right]^{\prime}$.

Proof of Proposition 4.1. The condition (4.12) is simply the union of those imposed in Theorem 3.5 and by Newey (1997) to obtain $\sup _{x \in \mathfrak{X}}|\hat{d}(x)-d(x)|=o_{p}(1)$. The proof of Theorem 1 in Newey (1997) implies $\left\|n^{-1} P^{\prime} P-\mathbb{E}\left(P_{J}\left(x_{i}\right) P_{J}\left(x_{i}\right)^{\prime}\right)\right\|=\mathscr{O}_{p}\left(\zeta(J) J^{\frac{1}{2}} / n^{\frac{1}{2}}\right)$ and $\left\|P^{\prime} v\right\|=\mathscr{O}_{p}\left((J n)^{\frac{1}{2}}\right)$. Together with (4.6) these imply that (3.4) holds under (4.12), because in the notation of (3.4) we have $M_{n}=P, \epsilon=v$ and $g_{n}=n^{-1} P^{\prime} v$ in this proposition.

Assumption A* ${ }^{*}$ (Robinson $\left.(2005,2010)\right)$ Define $\varphi=\left(1+\phi\left(t_{1}\right)\right)\left(\phi\left(t_{1}\right)-\phi\left(t_{2}\right)\right)^{-1}$ where $\left[t_{1}, t_{2}\right]$ is an interval on which $f(t)$ is bounded away from zero, and $\varkappa=1+2^{\frac{1}{2}} \bumpeq 2.414$, The following conditions hold:

- The $v_{i}$ are iid with zero mean, unit variance and differentiable pdf $f(t)$.

- $\phi_{\ell}(t)=\phi_{t}^{\ell}$ with $\phi(t)$ strictly increasing and thrice differentiable such that for some $\kappa \geq 0$ and $K<\infty,|\phi(t)| \leq 1+|t|^{\kappa}$ and $\left|\phi_{t}(t)\right|+\left|\phi_{t t}(t)\right|+\left|\phi_{t t t}(t)\right| \leq C\left(1+|\phi(t)|^{K}\right)$.

- $J \rightarrow \infty$ as $n \rightarrow \infty$, and either (i) $\kappa=0, \mathbb{E}\left(v_{i}^{4}\right)<\infty$, and

$$
\varliminf_{n \rightarrow \infty}(\log n / J)>8\{\log \varkappa+\max (\log \varphi, 0)\} \bumpeq 7.05+8 \max (\log \varphi, 0)
$$


or (ii) $\kappa>0$, for some $\omega>0$ the moment generating function $\mathbb{E}\left(\exp \left(u\left|v_{i}\right|^{\omega}\right)\right)$ exists for some $u>0$, and

$$
\varliminf_{n \rightarrow \infty}(\log n / J \log J) \geq \max \left\{8 \kappa \omega^{-1}, 4 \kappa(\omega+1) \omega^{-1}\right\}
$$

or (iii) $\kappa>0, v_{i}$ is almost surely bounded, and

$$
\varliminf_{n \rightarrow \infty}(\log n / J \log J) \geq 4 \kappa
$$

- Define $\rho_{v J}$ as $C J$ if $v=0,(C J)^{v J / \omega}$ if $v>0$ and Assumption $\mathrm{A}^{*}$ (ii) holds and $C^{J}$, if $v>0$ and Assumption $\mathrm{A}^{*}$ (iii) holds. Then

$$
\frac{1}{J}+\frac{1}{\mathfrak{t}(J)}+\frac{J^{\frac{1}{2}} \rho_{4 \kappa J}^{\frac{1}{2}}\left(\rho_{2 \kappa J} / \mathfrak{t}(J)+1\right)}{n^{\frac{1}{2}}} \longrightarrow 0 \text { as } n \rightarrow \infty .
$$

Proof of Proposition 5.1. The proof follows straightforwardly because as in Newey (1988) we can use $e_{i}=y_{n}-X_{n} \hat{\beta}_{O L S}$ and prove the result with $e_{i}$ replaced by $u_{i}$. Then $\mathbb{E}\left\|\bar{W}^{\prime(J)} u\right\|^{2}=$ $\sum_{i=1}^{n} \mathbb{E}\left\|\Phi^{(J)}\left(v_{i}\right)\right\|^{2}=\mathscr{O}_{p}\left(n \rho_{2 \kappa J}\right)$ by (A.37) of Robinson (2010) (note that we do not have the $t_{i i n}$ factor that arises in his SAR setup), whence $\left\|\bar{W}^{\prime(J)} u\right\|=\mathscr{O}_{p}\left(n^{\frac{1}{2}} \rho_{2 \kappa J}^{\frac{1}{2}}\right)$ by Markov's inequality. Similarly by Lemma 10 of Robinson $(2005)\left\|W^{(J)}-\mathbb{E}\left\{\Phi^{(J)}\left(v_{i}\right) \Phi^{(J)}\left(v_{i}\right)\right\}\right\|=$ $\mathscr{O}_{p}\left(J^{\frac{1}{2}} \rho_{4 \kappa J}^{\frac{1}{2}} / n^{\frac{1}{2}}\right)$. Now the conditions of the Proposition ensure that (3.4) and (3.7) are satisfied.

\section{B Technical lemmas}

Lemma B.1. Let the conditions of Theorem 3.3 hold. Conditional on elements of $M_{n}$, $\mathbb{E}\left(\epsilon^{\prime} \mathcal{M}_{n} \epsilon-q\right)=0$ and $\operatorname{var}\left(\epsilon^{\prime} \mathcal{M}_{n} \epsilon\right) / 2 q \stackrel{p}{\longrightarrow} 1$.

Proof. The first part follows from $\mathbb{E}\left(\epsilon^{\prime} \mathcal{M}_{n} \epsilon\right)=\operatorname{tr} \mathcal{M}_{n}=q$, by symmetry, idempotency and rank $q$ of $\mathcal{M}_{n}$. These properties also imply $\operatorname{var}\left(\epsilon^{\prime} \mathcal{M}_{n} \epsilon\right)=\left(\mu_{4}-3\right) \sum_{r} \mathfrak{m}_{r r, n}^{2}+\operatorname{tr}\left(\mathcal{M}_{n} \mathcal{M}_{n}^{\prime}\right)+$ $\operatorname{tr}\left(\mathcal{M}_{n}^{2}\right)=\mathscr{O}_{p}\left(n^{-1} s^{2} m^{4}\right)+2 q$, by (A.29), so $\operatorname{var}\left(\epsilon^{\prime} \mathcal{M}_{n} \epsilon\right) / 2 q=\mathscr{O}_{p}\left(q^{-1} n^{-1} s^{2} m^{4}\right)+1=$ $o_{p}(1)+1$, by $(3.7)$. 
Lemma B.2. Under the conditions of Theorem 3.1 or Theorem 3.2,

$$
\left\|\hat{H}_{n}^{-1}\right\|=\mathscr{O}_{p}\left(\left\|H_{n}^{-1}\right\|\right)=\mathscr{O}_{p}\left(\left\|\left(n^{-1} M_{n}^{\prime} M_{n}\right)^{-1}\right\|\right)=\mathscr{O}_{p}\left(\left\{\underline{\underline{\lim }} \underline{\underline{\eta}} \underline{\left(L_{n}\right)}\right\}^{-1}\right)=\mathscr{O}_{p}(1) .
$$

A similar result holds for evaluation at $\tilde{\theta}$ and $\bar{\theta}$ satisfying $\left\|\Delta_{\theta_{0}}^{\bar{\theta}}\right\| \leq\left\|\Delta_{\theta_{0}}^{\hat{\theta}}\right\|$ or $\left\|\Delta_{\theta_{0}}^{\bar{\theta}}\right\| \leq$ $\left\|\Delta_{\theta_{0}}^{\tilde{\theta}}\right\|$. The result also implies that the norms of blocks of partitioned versions of these matrices are bounded.

Proof. See Lemma B.3 in Gupta and Robinson (2018). For square blocks, the last claim follows by Lemma B.4. For non-square blocks, it follows as in the proof of Lemma B.5 (see (B.2)).

Lemma B.3. Under the conditions of Theorem 3.1 or Theorem 3.2,

$$
\left\|\hat{H}_{n}\right\|=\mathscr{O}_{p}\left(\left\|H_{n}\right\|\right)=\mathscr{O}_{p}\left(\left\|n^{-1} M_{n}^{\prime} M_{n}\right\|\right)=\mathscr{O}_{p}\left(\varlimsup_{n \rightarrow \infty} \bar{\eta}\left(L_{n}\right)\right)=\mathscr{O}_{p}(1) .
$$

A similar result holds for evaluation at $\tilde{\theta}$ and $\bar{\theta}$ satisfying $\left\|\Delta_{\theta_{0}}^{\bar{\theta}}\right\| \leq\left\|\Delta_{\theta_{0}}^{\hat{\theta}}\right\|$ or $\left\|\Delta_{\theta_{0}}^{\bar{\theta}}\right\| \leq$ $\left\|\Delta_{\theta_{0}}^{\tilde{\theta}}\right\|$. The result also implies that the norms of blocks of partitioned versions of these matrices are bounded.

Proof. Similar to proof of Lemma B.2.

Lemma B.4. Consider a square matrix $A_{n}$. Suppose that $\left\{\underline{\eta}\left(A_{n}\right)\right\}^{-1}+\bar{\eta}\left(A_{n}\right)=\mathscr{O}_{p}(1)$. Let $A_{n}^{\mathcal{S}}$ be any diagonal square sub-matrix of $A_{n}$. Then $\left\{\underline{\eta}\left(A_{n}^{\mathcal{S}}\right)\right\}^{-1}+\bar{\eta}\left(A_{n}^{\mathcal{S}}\right)=\mathscr{O}_{p}(1)$.

Proof. Follows easily by appropriate choice of $x_{n}$ in quadratic form $x_{n}^{\prime} A_{n} x_{n}$.

Lemma B.5. Under the conditions of Theorem 3.1 or Theorem 3.2, $\left\|\mathcal{H}_{n}\right\|=\mathscr{O}_{p}(1)$.

Proof. By elementary norm inequalities and properties

$$
\left\|\mathcal{H}_{n}\right\| \leq\left\|\mathcal{Z}^{\bar{H}}\right\|^{2}\left\|\hat{H}_{n}^{11}\right\|
$$

For the first factor on the RHS of (B.1), note that $\left\|\mathcal{Z}^{\bar{H}}\right\| \leq\left\|I_{q}\right\|+\left\|\bar{H}_{22, n}^{-1}\right\|\left\|\bar{H}_{21, n}\right\|$. Also $\bar{H}_{21, n}=I_{1, n} \bar{H}_{n} I_{2, n}$ where $I_{1, n}=\left[0_{(s-q) \times q}, I_{s-q}\right]$ and $I_{2, n}=\left[I_{q}, 0_{q \times(s-q)}\right]^{\prime}$. Clearly $\left\|I_{1, n}\right\|$, $\left\|I_{2, n}\right\| \leq 1$. Then

$$
\left\|\bar{H}_{21, n}\right\| \leq\left\|I_{1, n}\right\|\left\|I_{2, n}\right\|\left\|\bar{H}_{n}\right\| \leq\left\|\bar{H}_{n}\right\|=\mathscr{O}_{p}(1),
$$


by Lemma B.3. By the partitioned inverse formula, we have $\bar{H}_{22, n}^{-1}=\bar{H}_{n}^{22}-\bar{H}_{n}^{21}\left(\bar{H}_{n}^{22}\right)^{-1} \bar{H}_{n}^{12}$ implying that $\left\|\bar{H}_{22, n}^{-1}\right\| \leq\left\|\bar{H}_{n}^{22}\right\|+\left\|\bar{H}_{n}^{21}\right\|^{2}\left\|\left(\bar{H}_{n}^{22}\right)^{-1}\right\|$. Since eigenvalues of diagonal submatrices fall within the same bounds as those of the original matrix (see Lemma B.4) $\left\|\left(\bar{H}_{n}^{22}\right)^{-1}\right\|=\left\{\underline{\eta}\left(\bar{H}_{n}^{22}\right)\right\}^{-1}=\mathscr{O}_{p}(1)$. Arguments similar to those used for showing (B.2) allow us to conclude that $\left\|\bar{H}_{n}^{22}\right\|$ and $\left\|\bar{H}_{n}^{21}\right\|$ are $\mathscr{O}_{p}(1)$ using Lemma B.3. It follows that the first factor on the RHS of (B.1) is $\mathscr{O}_{p}(1)$. The second factor is also $\mathscr{O}_{p}(1)$ by Lemmas B.2 and B.4.

Lemma B.6. Under the conditions of Theorem 3.1 or Theorem 3.2, $\left\|\mathcal{Z}^{n^{-1} M^{\prime} M}\right\|+\left\|n^{-1} M_{n}^{\prime} M_{n}\right\|=\mathscr{O}_{p}(1)$.

Proof. The LHS is bounded by $\left\|\Delta_{\mathcal{Z}^{L}}^{\mathcal{Z}^{n^{-1} M^{\prime} M}}\right\|+\left\|\Delta_{L}^{n^{-1} M^{\prime} M}\right\|+\left\|\mathcal{Z}^{L}\right\|+\left\|L_{n}\right\|$. The last two terms are are $\mathscr{O}(1)$ by Assumption 2 and the techniques used in the proof of Lemma B.5,

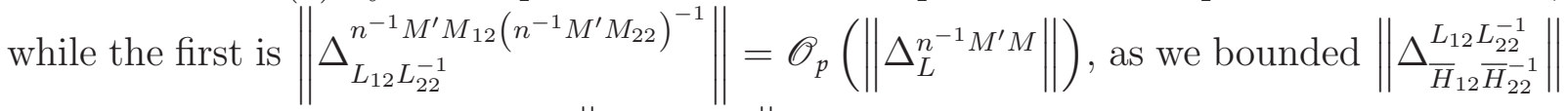
in the proof of Theorem 3.1. As $\left\|\Delta_{L}^{n^{-1} M^{\prime} M}\right\|=o_{p}(1)$ by (3.4), the lemma is proved. 


\section{References}

Ackerberg, D., Chen, X., Hahn, J., (2012). A practical asymptotic variance estimator for two-step semiparametric estimators. Review of Economics and Statistics 94, 481-498.

Ahmad, I., Leelahanon, S., Li, Q., (2005). Efficient estimation of a semiparametric partially linear varying coefficient model. Annals of Statistics 33, 258-283.

Andrews, D. W. K. (1985). A zero-one result for the least squares estimator. Econometric Theory 1, 85-96.

Andrews, D. W. K. (1991). Asymptotic normality of series estimators for nonparametric and semiparametric regression models. Econometrica 59, 307-345.

Andrews, D. W. K. (1994). Asymptotics for semiparametric econometric models via stochastic equicontinuity. Econometrica 62, 43-72.

Belloni, A., Chernozhukov, V., Chetverikov, D., Kato, K., (2015). Some new asymptotic theory for least squares series: Pointwise and uniform results. Journal of Econometrics 186, 345-366.

Beran, R. (1976). Adaptive estimates for autoregressive processes. Annals of the Institute of Statistical Mathematics 28, 77-89.

Berk, K. N. (1974). Consistent autoregressive spectral estimates. Annals of Statistics 2, 489-502.

Case, A. C. (1991). Spatial patterns in household demand. Econometrica 59, 953-965.

Case, A. C. (1992). Neighborhood influence and technological change. Regional Science and Urban Economics 22, 491-508.

Chen, X., Pouzo D., (2015). Sieve Wald and QLR inferences on semi/nonparametric conditional moment models. Econometrica 83, 1013-1079.

Cliff, A. D., Ord, J. K., (1973). Spatial Autocorrelation. London: Pion.

de Jong, R. M., Bierens, H. J., (1994). On the limit behavior of a chi-square type test if the number of conditional moments tested approaches infinity. Econometric Theory 10, 70-90. 
Donald, S. G., Imbens, G. W., Newey, W. K., (2003). Empirical likelihood estimation and consistent tests with conditional moment restrictions. Journal of Econometrics 117, $55-93$.

Eubank, R. L., Spiegelman, C. H.,(1990). Testing the goodness of fit of a linear model via nonparametric regression techniques. Journal of the American Statistical Association 85, $387-392$.

Fan, Y., Li. Q., (1996). Consistent model specification tests: Omitted variables and semiparametric functional forms. Econometrica 64, 865-890.

Gallant, R., Nychka, D. W., (1987). Semi-nonparametric maximum likelihood estimation. Econometrica 55, 363-390.

Gupta, A., Robinson, P. M., (2015). Inference on higher-order spatial autoregressive models with increasingly many parameters. Journal of Econometrics 186, 19-31.

Gupta, A., Robinson, P. M., (2018). Pseudo maximum likelihood estimation of higher-order spatial autoregressive models with increasing dimension. Journal of Econometrics 202, 92-107.

Gurmu, S., Rilstone, P., Stern, S., (1999). Semiparametric estimation of count regression models. Journal of Econometrics 88, 123-150.

Hong, Y., White, H., (1995). Consistent specification testing via nonparametric series regression. Econometrica 63, 1133-1159.

Huber, P. J. (1973). Robust regression: Asymptotics, conjectures and Monte Carlo. Annals of Statistics 1, 799-821.

Jenish, N. (2016). Spatial semiparametric model with endogenous regressors. Econometric Theory 32, 714-739.

Kelejian, H. H., Prucha, I. R., (1998). A generalized spatial two-stage least squares procedure for estimating a spatial autoregressive model with autoregressive disturbances. The Journal of Real Estate Finance and Economics 17, 99-121.

Lavergne, P., Vuong, Q., (2000). Nonparametric significance testing. Econometric Theory $16,576-601$. 
Le Cam, L. (1960). Locally asymptotically normal families of distributions. University of California publications in statistics 3, 37-98.

Lee, J., Robinson, P. M., (2016). Series estimation under cross-sectional dependence. Journal of Econometrics 190, 1-17.

Lee, L. F. (2002). Consistency and efficiency of least squares estimation for mixed regressive, spatial autoregressive models. Econometric Theory 18, 252-277.

Lee, L. F. (2004). Asymptotic distributions of quasi-maximum likelihood estimators for spatial autoregressive models. Econometrica 72, 1899-1925.

Lewbel, A. (2008). Engel curve. The New Palgrave Dictionary of Economics. Second Edition. Eds. Steven N. Durlauf and Lawrence E. Blume. Palgrave Macmillan.

Lorentz, G. G. (1986). Approximation of Functions. Chelsea.

Newey, W. K. (1988). Adaptive estimation of regression models via moment restrictions. Journal of Econometrics 38, 301-339.

Newey, W. K. (1997). Convergence rates and asymptotic normality for series estimators. Journal of Econometrics 79, 147-168.

Portnoy, S. (1984). Asymptotic behavior of $M$-estimators of $p$ regression parameters when $p^{2} / n$ is large. I. Consistency. Annals of Statistics 12, 1298-1309.

Portnoy, S. (1985). Asymptotic behavior of $M$-estimators of $p$ regression parameters when $p^{2} / n$ is large; II. Normal approximation. Annals of Statistics 13, 1403-1417.

Robinson, P. M. (1979). Distributed lag approximation to linear time-invariant systems. Annals of Statistics 7, 507-515.

Robinson, P. M. (1988). Root-n-consistent semiparametric regression. Econometrica 56, 931-954.

Robinson, P. M. (2003). Denis Sargan: Some perspectives. Econometric Theory 19, 481494.

Robinson, P. M. (2005). Efficiency improvements in inference on stationary and nonstationary fractional time series. Annals of Statistics 33, 1800-1842. 
Robinson, P. M. (2010). Efficient estimation of the semiparametric spatial autoregressive model. Journal of Econometrics 157, 6-17.

Scott, D. J. (1973). Central limit theorems for martingales and for processes with stationary increments using a Skorokhod representation approach. Advances in Applied Probability 5, 119-137.

Shen, X., Shi, J., (2005). Sieve likelihood ratio inference on general parameter space. Science in China Series A 48,67-78.

Su, L., Jin, S., (2010). Profile quasi-maximum likelihood estimation of partially linear spatial autoregressive models. Journal of Econometrics 15\%, 18-33.

van der Vaart, A. (2002). The statistical work of Lucien Le Cam. Annals of Statistics 30, 631-682.

Wooldridge, J. M. (1992). A test for functional form against nonparametric alternatives. Econometric Theory 8, 452-475.

Yatchew, A. J. (1992). Nonparametric regression tests based on least squares. Econometric Theory 8, 435-451.

Yatchew, A. J. (2000). Scale economies in electricity distribution: a semiparametric analysis. Journal of Applied Econometrics 210, 187-210.

Yatchew, A. J. (2003). Semiparametric Regression for the Applied Econometrician. Cambridge University Press.

Zheng, J. X. (1996). A consistent test of functional form via nonparametric estimation techniques. Journal of Econometrics 75, 263 - 289. 